Algebraic $8 \mathcal{G}$ Geometric $\mathcal{T}$ opology

Volume 4 (2004) 1045-1081

Published: 6 November 2004

ATG

\title{
sl(3) link homology
}

\author{
Mikhail Khovanov
}

\begin{abstract}
We define a bigraded homology theory whose Euler characteristic is the quantum $\mathrm{sl}(3)$ link invariant.
\end{abstract}

AMS Classification 81R50, 57M27; 18G60

Keywords Knot, link, homology, quantum invariant, sl(3)

\section{Introduction}

To each simple Lie algebra $\mathfrak{g}$ there is associated a polynomial invariant $\langle L\rangle$ of oriented links in $\mathbb{R}^{3}$ with components labelled by irreducible finite-dimensional representation of $\mathfrak{g}$, see [13, 9] and references therein. Suitably normalized, the invariant takes values in $\mathbb{Z}\left[q, q^{-1}\right]$.

We conjecture that, for each simply-laced $\mathfrak{g}$, the invariant $\langle L\rangle$ admits a categorification: there exists a bigraded homology theory of labelled links

$$
\mathcal{H}_{\mathfrak{g}}(L)=\oplus_{i, j \in \mathbb{Z}} \mathcal{H}_{\mathfrak{g}}^{i, j}(L)
$$

with $\langle L\rangle$ as the Euler characteristic,

$$
\langle L\rangle=\sum_{i, j}(-1)^{i} q^{j} \operatorname{rk}\left(\mathcal{H}_{\mathfrak{g}}^{i, j}(L)\right) .
$$

In the first nontrivial case - when $\mathfrak{g}$ is $\mathfrak{s l}(2)$ and all components of $L$ are labelled by its defining representation - this theory was constructed in [6]. An oriented surface cobordism $S \subset \mathbb{R}^{4}$ between links $L_{1}$ and $L_{2}$ induces a homomorphism $\mathcal{H}(S): \mathcal{H}\left(L_{1}\right) \rightarrow \mathcal{H}\left(L_{2}\right)$, well-defined up to overall minus sign, see [3] [5]. These homomorphisms fit together into a functor from the category of link cobordisms to the category of bigraded abelian groups and group homomorphisms (with identifications $f=-f$ for each homomorphism). Likewise, the theory $\mathcal{H}_{\mathfrak{g}}$ should extend to a functor from the category of oriented framed cobordisms between framed links to the category of bigraded abelian groups and group homomorphisms, with \pm identification. For $\mathfrak{s l}(2)$ and its defining representation the framing can be hidden, but not, apparently, in the general 
case. Components of links and link cobordisms should be labelled by irreducible representations of $\mathfrak{g}$.

The homology of the unknot labelled by an irreducible representation $V$ should be a commutative Frobenius algebra of dimension $\operatorname{dim}(V)$, since the category of link cobordisms contains a subcategory equivalent to the category of 2-cobordisms between 1-manifolds (not embedded anywhere). This algebra should be, in addition, graded, and its graded dimension should equal the quantum dimension of $V$. When $V$ is the $k$-th exterior power of the defining representation of $\mathfrak{s l}(n)$, this algebra is going to be the cohomology of the Grassmannian of $k$-dimensional subspaces of $\mathbb{C}^{n}$.

When $\mathfrak{g}=\mathfrak{s l}(2)$ and components of $L$ are labelled by arbitrary $\mathfrak{s l}(2)$ representations, the invariant $\langle L\rangle$ is the colored Jones polynomial. Its categorification is sketched in [4].

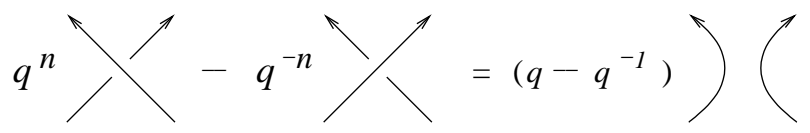

Figure 1: Quantum $\mathfrak{s l}(n)$ skein formula

When $\mathfrak{g}=\mathfrak{s l}(n)$ and each components of $L$ is labelled either by the defining representation $V$ or its dual, the invariant is determined by the skein relation in Figure 1. If we introduce a second variable $p=q^{n}$, the skein relation gives rise to the HOMFLY polynomial, a 2-variable polynomial invariant of oriented links [2]. We do not believe in a triply-graded homology theory categorifying the HOMFLY polynomial. Instead, for each $n \geq 0$ there should exist a bigraded theory categorifying the $\left(q, q^{n}\right)$ specialization of HOMFLY. For $n=0$ such theory was constructed by Peter Ozsváth and Zoltán Szabó [11] and, independently, by Jacob Rasmussen [12]. For $n=1$ the polynomial invariant as well as the homology theory is trivial. For $n=2$ the invariant is the Jones polynomial and was categorified in [6]. In this paper we construct link homology in the $n=3$ case. In the sequel we will extend this invariant to tangles and tangle cobordisms.

We use Greg Kuperberg's approach to the quantum $\mathfrak{s l}(3)$ link invariant 8 . He described the representation theory of $U_{q}(\mathfrak{g})$, for each rank two simple Lie algebra $\mathfrak{g}$, via a calculus of planar trivalent graphs, called webs. Each edge of a web is labelled by one of the two fundamental representation of $U_{q}(\mathfrak{g})$, and a trivalent vertex denotes a vector in the invariant space of a triple tensor product. A web with boundary gives a vector in the invariant space of a tensor 
product of fundamental representations. A closed web evaluates to a polynomial in $q^{ \pm \frac{1}{2}}$ with integer coefficients if $\mathfrak{g}=\mathfrak{s l}(3)$; to a polynomial in $q^{ \pm 1}$ with integer coefficients, if $\mathfrak{g}=\mathfrak{s o}(5)$; and to a rational function of $q$ if $\mathfrak{g}=G_{2}$ (it is not immediately clear from [8] that $G_{2}$ webs always evaluate to polynomials).

Among $\mathfrak{s l}(3), \mathfrak{s o}(5)$, and $G_{2}$, the three simple Lie algebras or rank 2, only $\mathfrak{s l}(3)$ is simply-laced. This property seems to relate to positivity of the Kuperberg calculus for $\mathfrak{s l}(3)$. Namely, in the $\mathfrak{s l}(3)$ case, closed webs evaluate to Laurent polynomials in $q$ with non-negative coefficients, after changing Kuperberg's $q^{\frac{1}{2}}$ to $-q$. No such positivity is at hand for the other two calculi.

To compute the $\mathfrak{s l}(3)$ invariant of a link $L$ choose its plane projection $D$ and flatten each crossing in two possible ways, as in Figure 9. This action produces $2^{n}$ closed webs, where $n$ is the number of crossings of $D$. The invariant of $L$ is the sum of these webs' evaluations weighted by powers of $q$ and -1 , as explained in Figure 10. Webs are evaluated recursively, through Figure 7 reductions (where $[3]=q^{2}+1+q^{-2}$ and $[2]=q+q^{-1}$ ). Each transformation either reduces the number of vertices or removes a loop. Since the formulas have no minus signs in them, the evaluation $\langle\Gamma\rangle$ of any closed web $\Gamma$ has non-negative integer coefficients and lies in $\mathbb{Z}_{+}\left[q, q^{-1}\right]$.

Positivity of $\langle\Gamma\rangle$ is a hint that its categorification is just a graded abelian group, rather than a complex of graded abelian groups. Since $\langle\Gamma\rangle=\sum a_{j} q^{j}$ for some non-negative integers $a_{j}$, the categorification (or homology) $\mathcal{F}(\Gamma)$ of $\langle\Gamma\rangle$ should be a graded abelian group $\underset{j \in \mathbb{Z}}{\oplus} F_{j}$ with $\operatorname{rank}\left(F_{j}\right)=a_{j}$.

Homology $\mathcal{F}(\Gamma)$ for different $\Gamma$ must be related. Namely, we need to have maps between homologies of any two webs that differ as in the R.H.S. of Figure 10. With these maps at hand we could set up a commutative cube of web homologies and maps, form the total complex of this cube and take its homology, following the approach of [6]. How to define these maps? In the $\mathfrak{s l}(2)$ homology theory of links maps come from cobordisms. We want to have maps between homology of webs, and expect those to come from web cobordisms, which we will call foams. Locally, a foam should be homeomorphic to a neighbourhood of a point on the web times an interval. Since webs have singular points (vertices) with neighbourhoods homeomorphic to letter $Y$, foams will have singular arcs near which, locally, the foam is $Y \times[0,1]$. An example of a foam is depicted in Figure 2. Another example is the identity cobordism $\Gamma \times[0,1]$.

To each foam $U$ with boundaries $\Gamma_{1}$ and $\Gamma_{2}$ we want to associate a homomorphism $\mathcal{F}(U): \mathcal{F}\left(\Gamma_{1}\right) \longrightarrow \mathcal{F}\left(\Gamma_{2}\right)$. 

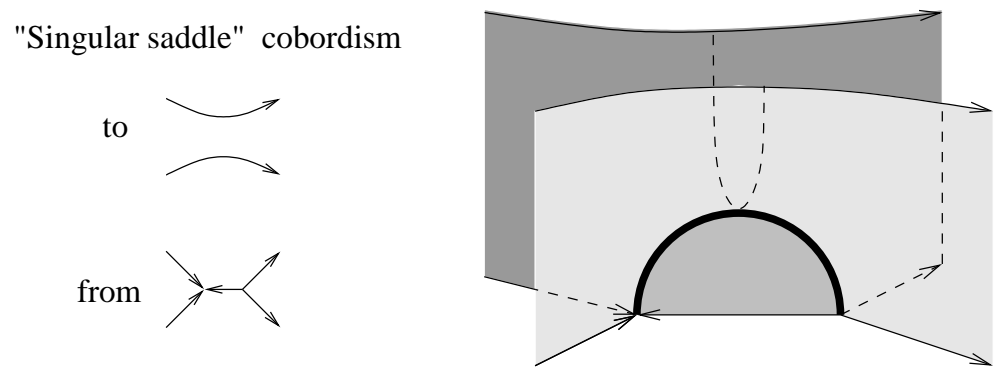

Figure 2: Only the nontrivial portion of the cobordism is indicated

For the moment let us put foams aside and return to webs. The unknot evaluates to [3] $=q^{2}+1+q^{-2}$. Therefore, to the unknot we should associate a graded Frobenius algebra $\mathcal{A}$ which has $\mathbb{Z}$ in degrees $-2,0,2$. Our only choice is to take $\mathcal{A}$ to be the ring $\mathbb{Z}[X] / X^{3}$, the cohomology ring of $\mathbb{P}^{2}$. We shift the degrees of this cohomology ring down by 2 to balance them around degree 0 .

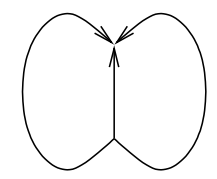

Figure 3: Theta web $\Theta$

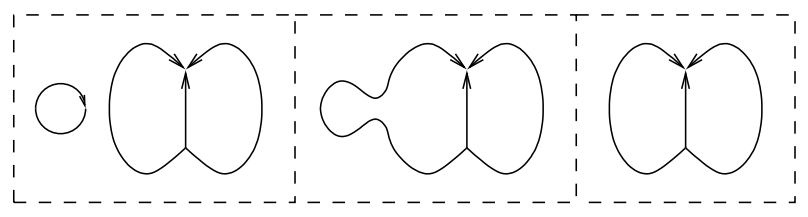

Figure 4: Web cobordism cross-sections: a circle merges into an edge of $\Theta$

The theta web in Figure 3 is the simplest web with trivalent vertices. It evaluates to [3][2], and we want to assign to $\Theta$ a graded abelian group $\mathcal{F}(\Theta)$ of graded rank [3][2]. For each edge of $\Theta$ there is a web cobordism of merging a circle into the edge, depicted in Figure 4. This cobordism should induce a map of abelian groups

$$
\mathcal{A} \otimes \mathcal{F}(\Theta) \longrightarrow \mathcal{F}(\Theta)
$$

and make $\mathcal{F}(\Theta)$ into an $\mathcal{A}$-module. The three $\mathcal{A}$-module structures on $\mathcal{F}(\Theta)$ should commute and make $\mathcal{F}(\Theta)$ into an $\mathcal{A}^{\otimes 3}$-module. 
Consider the cohomology ring of the full flag variety of $\mathbb{C}^{3}$,

$$
F l_{3} \stackrel{\text { def }}{=}\left\{\left(V_{1}, V_{2}\right) \mid V_{1} \subset V_{2} \subset \mathbb{C}^{3}, \operatorname{dim} V_{i}=i\right\} .
$$

Choose a hermitian inner product on $\mathbb{C}^{3}$. The flag variety is homeomorphic to the space of triples of pairwise orthogonal complex lines in $\mathbb{C}^{3}$ :

$$
F l_{3} \cong\left\{\left(W_{1}, W_{2}, W_{3}\right) \mid W_{i} \subset \mathbb{C}^{3}, \operatorname{dim} W_{i}=1, \quad W_{i} \perp W_{j}, i \neq j\right\} .
$$

This homeomorphism defines an embedding of $\mathrm{Fl}_{3}$ into $\mathbb{P}^{2} \times \mathbb{P}^{2} \times \mathbb{P}^{2}$. The induced map on cohomology makes $\mathrm{H}^{*}\left(F l_{3}, \mathbb{Z}\right)$ into an $\mathcal{A}^{\otimes 3}$-module. Note that $q^{3}[3][2]$ is the graded dimension of $\mathrm{H}^{*}\left(F l_{3}, \mathbb{Z}\right)$. We define $\mathcal{F}(\Theta)$ as the integral cohomology ring of $\mathrm{Fl}_{3}$ with the grading shifted down by 3 :

$$
\mathcal{F}(\Theta)=\mathrm{H}^{*}\left(F l_{3}, \mathbb{Z}\right)\{-3\} .
$$

A naive generalization of this approach to $\mathcal{F}(\Gamma)$ works for digon webs, that is, webs which can be obtained from a circle by adding digon faces, see Section 6 , A digon web is shown in Figure 5 left. To define $\mathcal{F}(\Gamma)$ already for the "cube" web on Figure 5 requires a different method.
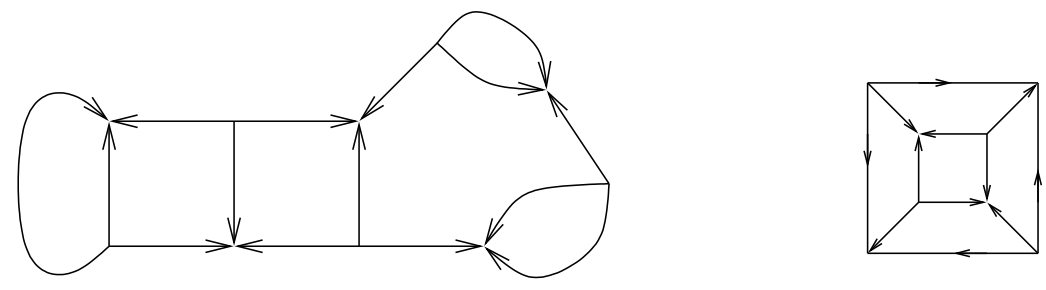

Figure 5: A digon web and the cube web

In Section 3 we define homology $\mathcal{F}(\Gamma)$ for any web $\Gamma$. A foam $U$ should induce a map between homology groups of its boundaries. The homology of the empty web should be $\mathbb{Z}$, and if $U$ has no boundaries (we call $U$ a closed foam), it should induce an endomorphism of $\mathbb{Z}$, that is, an integer. These integers (evaluations of closed foams) must be compatible with our assignments of $\mathcal{A}$ to the circle web and cohomology of the flag variety to the theta web. Compatibility requirements force us into a unique way to evaluate closed foams, explained in Section 3.2.

A foam which is a cobordism from the empty web to $\Gamma$ should define a homomorphism from $\mathbb{Z}$ to $\mathcal{F}(\Gamma)$, or, equivalently, an element of $\mathcal{F}(\Gamma)$. We define homology $\mathcal{F}(\Gamma)$ implicitly, as abelian group generated by foams from the empty web to $\Gamma$ and relations coming from required compatibility with evaluations of closed foams, see Section 3.3 The price of our implicit definition is in the 
work needed to establish skein relations for web homology. This is done in Section 3.4 .

Given a diagram $D$ of a link $L$, we arrange the graded abelian groups $\mathcal{F}\left(D_{f}\right)$, for all flattenings $f$ of $D$, into a complex $\mathcal{F}(D)$ of graded abelian groups, see Section 4. In Section [5 we prove

Theorem 1 If diagrams $D_{1}$ and $D_{2}$ are related by a Reidemeister move, the complexes $\mathcal{F}\left(D_{1}\right)$ and $\mathcal{F}\left(D_{2}\right)$ are chain homotopic.

Let $\mathcal{H}(D)=\underset{i, j \in \mathbb{Z}}{\oplus} \mathcal{H}^{i, j}(D)$ be the cohomology groups of $\mathcal{F}(D)$.

Corollary 1 Isomorphism classes of groups $\mathcal{H}^{i, j}(D)$ are invariants of $L$.

Proposition 1 The Euler characteristic of $\mathcal{H}(L)$ is the quantum $\mathfrak{s l}_{3}$ invariant:

$$
\langle L\rangle=\sum_{i, j}(-1)^{i} q^{j} \operatorname{rk}\left(\mathcal{H}^{i, j}(L)\right) .
$$

Let us briefly discuss whether the invariants defined in [6] and the present paper should be called homology or cohomology. We are building functors from the category of link cobordisms to the category of abelian groups. Homology is a covariant functor, cohomology a contravariant one. A link cobordism between two links can be viewed as a morphism in either direction. In other words, the category of link cobordisms has a contravariant involution, which restricts to the identity on objects. Composing with this involution turns any covariant functor into a contravariant one. This allows us to turn any homology theory of cobordisms into a cohomology theory and vice versa. Previously we used the term "cohomology," and in this paper switch to "homology," for balance. Differentials in our complexes increase the index by 1 , but this convention can be easily reversed.

A calculus of planar trivalent graphs that gives rise to quantum $\mathfrak{s l}(n)$ link invariants for any $n$ was developed by Murakami, Ohtsuki and Yamada 10, and further studied by Dongseok Kim [7]. This calculus has the same positivity flavor as Kuperberg's. We expect that to each such planar graph there is naturally associated a graded abelian group whose graded rank equals the polynomial invariant of the graph, and these groups can be assembled into complexes to produce quantum $\mathfrak{s l}(n)$ link homology. Cohomology rings of Grassmannians and partial flag varieties will play an essential role in this theory. 
Acknowledgments I am indebted to Greg Kuperberg for many illuminating discussions and valuable input. Section [ w was written jointly with Greg Kuperberg. Dror Bar-Natan's suggestion to try making this paper readable led to a rather long introduction. While writing this paper, I was partially supported by the NSF grant DMS-0104139 and daily intakes of grande white chocolate mocha at Starbucks.

\section{Planar trivalent graphs and the quantum sl(3) in- variant}

A closed web in the Kuperberg $\mathfrak{s l}(3)$ spider is a trivalent oriented graph in $\mathbb{R}^{2}$, possibly with verticeless loops, such that at each vertex the edges are either all oriented in or oriented out, see Figure 6. Any such graph is bipartite.

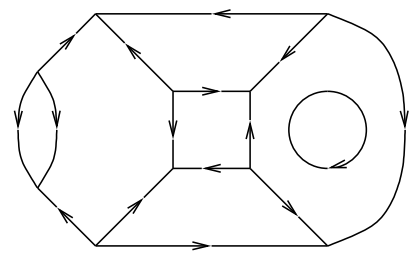

Figure 6: A closed web

To a web $\Gamma$ associate a Laurent polynomial $\langle\Gamma\rangle \in \mathbb{Z}\left[q, q^{-1}\right]$, the Kuperberg bracket of $\Gamma$, via the rules depicted in Figure [7] where [2] $=q+q^{-1}$ and $[3]=q^{2}+1+q^{-2}$.

These rules say that

(i) If $\Gamma$ is a disjoint union of $\Gamma_{1}$ and a loop, then

$$
\langle\Gamma\rangle=[3]\left\langle\Gamma_{1}\right\rangle
$$

(ii) If $\Gamma_{1}$ is obtained by deleting a digon face of $\Gamma$ then

$$
\langle\Gamma\rangle=[2]\left\langle\Gamma_{1}\right\rangle
$$

(iii) If $\Gamma_{1}, \Gamma_{2}$ are given by deleting pairs of opposite edges from a square face of $\Gamma$ then

$$
\langle\Gamma\rangle=\left\langle\Gamma_{1}\right\rangle+\left\langle\Gamma_{2}\right\rangle
$$




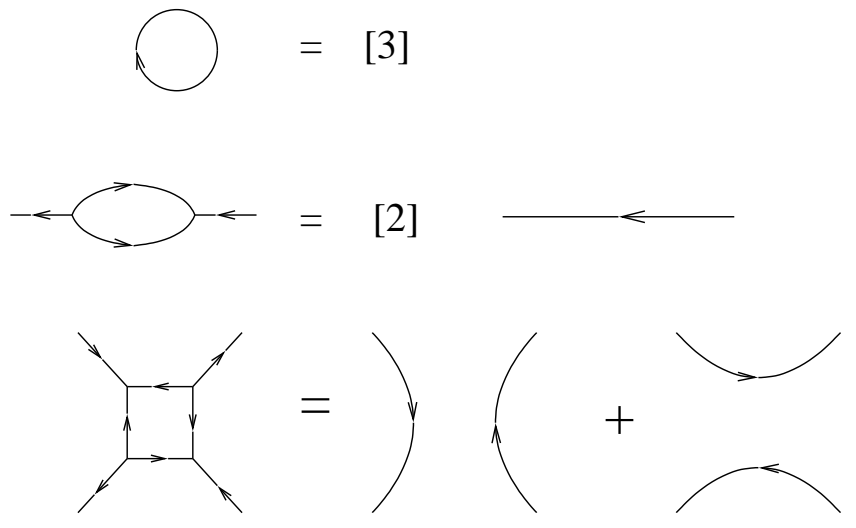

Figure 7: Web skein relations

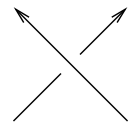

positive

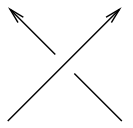

negative

Figure 8: Types of crossings

Since a closed web contains either a loop, or a digon face, or a square face, these rules determine the invariant. For a proof that they are consistent see Kuperberg [8].

Let $D$ be a generic planar projection of an oriented link $L$ and $n$ the number of crossings in $D$. We distinguish between positive and negative crossings, see Figure 8

For each crossing of $D$ consider two "flattenings" of this crossing as in Figure 9 Define $\langle D\rangle$, the bracket of $D$, as the linear combination of the brackets of all $2^{n}$ flattenings $D_{f}$ of $D$, via the rules in Figure 10.

$$
\langle D\rangle \stackrel{\text { def }}{=} \sum_{f \in\{0,1\}^{n}} b_{f}\left\langle D_{f}\right\rangle .
$$

The sum runs over all flattenings $f$ of $D$, and $b_{f}$ is plus or minus a power of $q$, see Figure 10.

Proposition $2\langle D\rangle$ is preserved by the Reidemeister moves. 


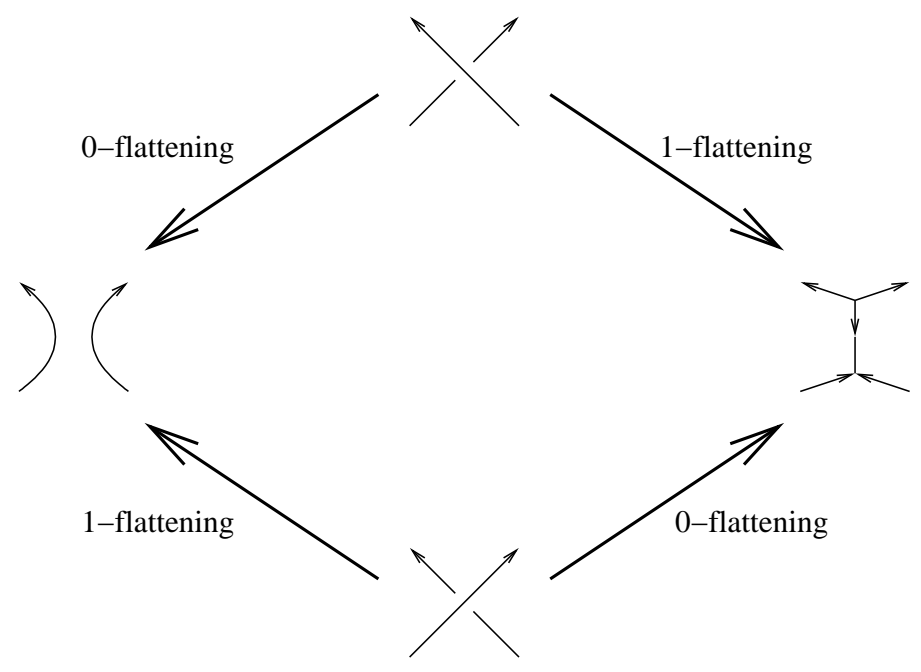

Figure 9: Flattenings
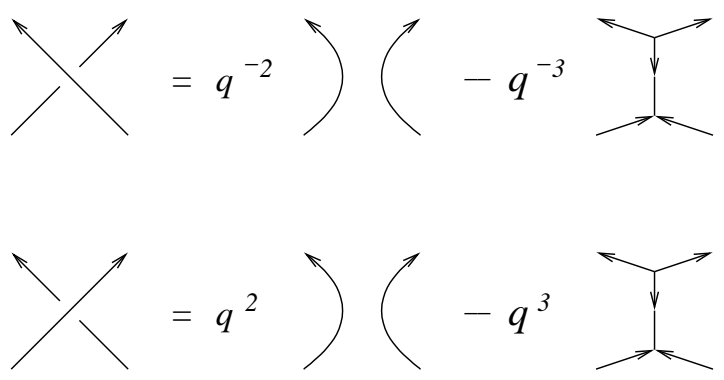

Figure 10: Decompositions of crossings

Thus, $\langle D\rangle$ is an invariant of an oriented link $L$, and will be denoted $\langle L\rangle$. This invariant of links is associated with the quantum group $U_{q}\left(\mathfrak{s l}_{3}\right)$ and its fundamental representations. Excluding rightmost terms from the equations in Figure 10 yields the $\mathfrak{s l}(3)$ specialization of the HOMFLY skein relation (Figure 11).

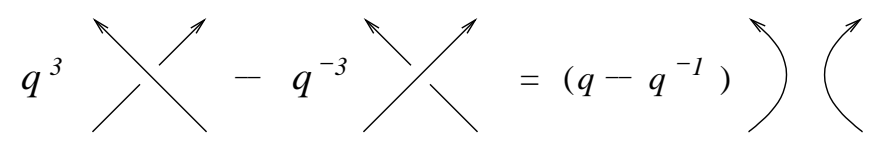

Figure 11: Quantum $\mathfrak{s l}(3)$ skein formula

$\langle L\rangle$ does not depend on the framing of $L$. Kuperberg [8] uses a slightly different, 
frame-dependent, normalization of this invariant.

\section{Foams}

\subsection{A (1+1)-dimensional TQFT with dots}

Let $\mathcal{A}=\mathbb{Z}[X] / X^{3}$. This ring is commutative Frobenius with the trace

$$
\varepsilon: \mathcal{A} \rightarrow \mathbb{Z}, \quad \varepsilon\left(X^{2}\right)=-1, \quad \varepsilon(X)=0, \quad \varepsilon(1)=0 .
$$

We make $\mathcal{A}$ into a graded ring, by $\operatorname{deg}(1)=-2, \operatorname{deg}(X)=0, \operatorname{deg}\left(X^{2}\right)=2$. Multiplication and comultiplication in $\mathcal{A}$ have degree 2 , the unit and trace maps have degree -2 . The comultiplication is the map $\mathcal{A} \rightarrow \mathcal{A} \otimes \mathcal{A}$ dual to the multiplication via the trace form,

$$
\begin{aligned}
\Delta(1) & =-1 \otimes X^{2}-X \otimes X-X^{2} \otimes 1, \\
\Delta(X) & =-X \otimes X^{2}-X^{2} \otimes X, \\
\Delta\left(X^{2}\right) & =-X^{2} \otimes X^{2} .
\end{aligned}
$$

$\mathcal{A}$ is the cohomology ring of the complex projective plane with the reverse complex orientation,

$$
\mathcal{A} \cong \mathrm{H}^{*}\left(\overline{\mathbb{P}^{2}}, \mathbb{Z}\right),
$$

the trace map being the evaluation on the fundamental 4-cycle. The grading of $\mathrm{H}^{*}\left(\overline{\mathbb{P}^{2}}, \mathbb{Z}\right)$ is shifted downward by 2 , to balance cohomology groups around degree 0 . Reversing orientation of $\mathbb{P}^{2}$ changes the trace map, not the multiplication. Note that $(\mathcal{A}, \varepsilon)$ and $(\mathcal{A},-\varepsilon)$ are not isomorphic as Frobenius algebras (but become isomorphic after adding $\sqrt{-1}$ to the ground ring $\mathbb{Z}$ ). Our choice of trace is essential for what follows.

The commutative Frobenius ring $\mathcal{A}$ gives rise to a functor from the category of 2-dimensional oriented cobordisms to the category of graded abelian groups. We denote this functor by $\mathcal{F}$. On objects $\mathcal{F}$ is given by $\mathcal{F}(M) \cong \mathcal{A}^{\otimes J}$ where $J$ is the set of components of a one-manifold $M$. To the "pants" cobordism from 2 circles to 1 circle $\mathcal{F}$ associates the multiplication map, etc. For more details see [1, Section 4.3], [6].

All our tensor products are over $\mathbb{Z}$ unless specified otherwise. Denote by $\{n\}$ the shift in the grading up by $n$.

The homomorphism $\mathcal{F}(S)$ associated with a cobordism $S$ has degree $-2 \chi(S)$ where $\chi$ is the Euler characteristic of $S$. Multiplication by $X$ increases the degree by 2 . 
A dot on a surface will denote multiplication by $X$. For instance, the annulus $S^{1} \times[0,1]$ is the identity cobordism from a circle to itself, and induces the identity map Id $: \mathcal{A} \rightarrow \mathcal{A}$. The same annulus with an added dot is the multiplication by $X$ endomorphism of $\mathcal{A}$. Figure 12 shows two other examples. The functor $\mathcal{F}$ applied to the "cup" morphism gives the unit map $\mathbb{Z} \rightarrow \mathcal{A}$, and applied to the "cup" with a dot, see Figure 12 left, produces the map $\mathbb{Z} \rightarrow \mathcal{A}$ which takes 1 to $X$. A twice dotted annulus is the multiplication by $X^{2}$ endomorphism of $\mathcal{A}$. Dots can move freely along a connected component of a surface. In the obvious way $\mathcal{F}$ extends to a functor from the category of oriented dotted $(1+1)$-cobordisms to the category of graded abelian groups.
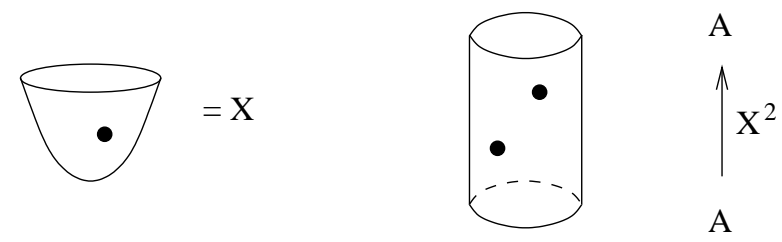

multiplication by $\mathrm{X}^{2}$

Figure 12: The meaning of dots

The degree formula

$$
\operatorname{deg}(\mathcal{F}(S))=-2 \chi(S)
$$

works for dotted cobordisms as well if by $\chi(S)$ we denote the Euler characteristic of $S$ with dots punctured out.

The map $\mathcal{F}(S)$ is zero in each of the following cases:

(i) a connected component of $S$ has genus 2 or more,

(ii) a connected component of $S$ has genus 1 and at least one dot,

(iii) a connected component of $S$ contains at least 3 dots.

Given any commutative Frobenius algebra $R$ with non-degenerate trace $\varepsilon$, choose a basis $\left\{x_{i}\right\}$ of $R$ and form the dual basis $\left\{y_{i}\right\}$ determined by $\varepsilon\left(x_{i} y_{j}\right)=$ $\delta_{i, j}$. Then we have a decomposition $x=\sum y_{i} \varepsilon\left(x_{i} x\right)$ for any $x \in R$. For $\mathcal{A}$ and the basis $\left(1, X, X^{2}\right)$ the dual basis is $\left(-X^{2},-X,-1\right)$. The decomposition

$$
-x=X^{2} \varepsilon(x)+X \varepsilon(X x)+\varepsilon\left(X^{2} x\right)
$$

has a geometric interpretation via a surgery on an annulus, see Figure 13.

If $S$ is a closed connected oriented surface, possibly decorated by dots, then $\mathcal{F}(S)=0$ unless 


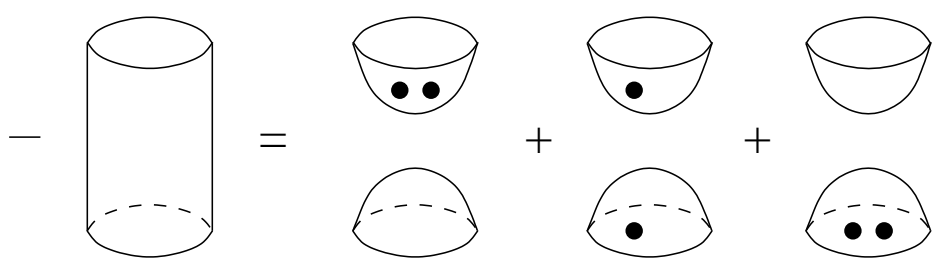

Figure 13: The surgery formula

(i) $S$ is a 2 -sphere decorated by 2 dots, then $\mathcal{F}(M)=-1$,

or

(ii) $\quad M$ is a dotless torus, then $\mathcal{F}(M)=3$.

The surgery formula implies the genus reduction formula in Figure 14.

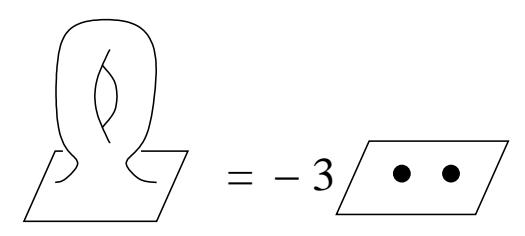

Figure 14: Genus reduction

\subsection{Pre-foams}

Definition Informally, a pre-foam is a 2-dimensional CW-complex with singular circles and some additional data. A point on a singular circle has a neighbourhood homeomorphic to the product of the letter $\mathrm{Y}$ and an interval, see Figure 15.

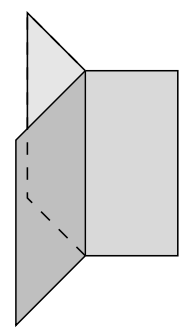

Figure 15: Singularities of a pre-foam 
Precisely, a pre-foam $U$ consists of

(i) an orientable smooth compact surface $U^{\prime}$ with connected components $U^{1}, U^{2}$, $\ldots, U^{r}$. The boundary components of $U^{\prime}$ are partitioned into triples (the set of boundary components is decomposed into a disjoint union of three-element sets). The three circles $C^{i}, C^{j}, C^{k}$ in each triple are identified via a pair of diffeomorphisms

$$
C^{i} \cong C^{j} \cong C^{k}
$$

We define the two-dimensional $\mathrm{CW}$-complex underlying $U$ as the quotient of $U^{\prime}$ by these identifications.

Thus, a triple $\left(C^{i}, C^{j}, C^{k}\right)$ of boundary components becomes a circle $C$ in the quotient $\mathrm{CW}$-complex $U^{\prime} / \sim$, called a singular circle. A singular circle has a neighbourhood diffeomorphic to the direct product of a circle and the letter $Y$.

We call the images of surfaces $U^{i}$ in the quotient CW-complex $U^{\prime} / \sim$ the facets of $U$. The image of the interior $U^{i} \backslash \partial\left(U^{i}\right)$ of $U^{i}$ in $U^{\prime} / \sim$ is an open connected orientable surface.

(ii) A cyclic ordering at each singular circle $C$ of the three boundary annuli of $U^{\prime}$ meeting at $C$ (note that there are two possible cyclic orderings at each $C$ ).

(iii) A number of dots (possibly none) marking each facet of $U$. A dot is allowed to move freely along the facet it belongs to, but can't cross a singular circle and move to another facet.

Example If $U^{\prime}$ is closed, the pre-foam $U=U^{\prime}$ is a closed surface decorated by dots.

Example Let $U^{\prime}$ be the disjoint union of three discs. Then $U$ has one singular circle, and is diffeomorphic to the 2 -sphere with added equatorial disc. Fix a cyclic ordering of facets with the equatorial disc followed by the northern hemisphere followed by the southern hemisphere followed by the equatorial disc. We call this pre-foam the theta-foam. If $a$ dots mark the equatorial disc, $b$ dots mark the northern hemisphere, and $c$ dots mark the southern hemisphere, we denote this pre-foam by $\theta(a, b, c)$. The pre-foams $\theta(a, b, c), \theta(b, c, a), \theta(c, a, b)$ are isomorphic. Isomorphism (or homeomorphism) of pre-foams is defined in the obvious way.

Evaluation To each pre-foam $U$ we assign an integer $\mathcal{F}(U)$, the evaluation of $U$, via the following rules:

(1) If $U$ is a closed surface (i.e. has no singular circles), possibly with dots, then $\mathcal{F}(U)$ is defined via the 2D TQFT constructed in section 3.1 
(2) $\mathcal{F}$ is multiplicative with respect to the disjoint union of pre-foams:

$$
\mathcal{F}\left(U_{1} \sqcup U_{2}\right)=\mathcal{F}\left(U_{1}\right) \mathcal{F}\left(U_{2}\right) .
$$

(3) Given a circle inside a facet of $U$, do a surgery on this circle, as in Figure 13 (on any circle wrapping once around the cylinder on the left hand side). Let $U_{1}, U_{2}, U_{3}$ be the resulting pre-foams (depicted on the right). Then

$$
-\mathcal{F}(U)=\mathcal{F}\left(U_{1}\right)+\mathcal{F}\left(U_{2}\right)+\mathcal{F}\left(U_{3}\right) .
$$

The singular circles of a pre-foam are disjoint from the circles on which we can do surgeries. A surgery circle should lie in the interior of a facet, and surgeries happen away from singular circles.

(4) The theta-foam evaluates as follows:

$$
\mathcal{F}(\theta(a, b, c))=\left\{\begin{array}{l}
0 \text { if } a+b+c \neq 3 \text { or } a=b=c=1 \\
1 \text { if }(a, b, c)=(0,1,2), \text { up to cyclic permutation, } \\
-1 \text { if }(a, b, c)=(0,2,1), \text { up to cyclic permutation }
\end{array}\right.
$$

$\theta(0,1,2)$

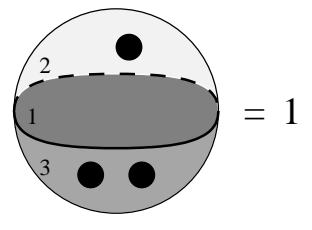

$\theta(2,1,0)$

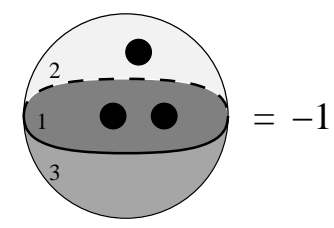

Figure 16: Theta-foam evaluations. The cyclic order of facets near the singular circle is $1 \rightarrow 2 \rightarrow 3 \rightarrow 1$.

Proposition 3 Rules (1)-(4) are consistent and uniquely determine $\mathcal{F}(U)$ for any pre-foam $U$.

Proof To show that the rules determine the evaluation, deform each singular circle $C$ of $U$ to three circles $C_{1}, C_{2}, C_{3}$ inside the three annuli of $U$ near $C$. Do a surgery on each of $C_{1}, C_{2}, C_{3}$. We get

$$
\mathcal{F}(U)=\sum_{i} a_{i} \mathcal{F}\left(U_{i}\right)
$$

where $a_{i} \in \mathbb{Z}$, and each foam $U_{i}$ is a disjoint union of closed orientable surfaces and theta-foams, possibly dotted. Rules (1),(2),(4) determine $\mathcal{F}\left(U_{i}\right)$. 
To prove consistency, we note that any evaluation via (1)-(4) requires separating each singular circle from the rest of $U$ and creating a theta-foam just for this circle. Functoriality of the functor $\mathcal{F}$ on dotted surfaces (non-singular prefoams) implies consistency of $\mathcal{F}(U)$ when $U$ has no singular circles. Therefore, it suffices to check consistency when $U$ is a theta-foam $\theta(a, b, c)$. We could either evaluate $\theta(a, b, c)$ following (4), or do a surgery on a circle of $\theta(a, b, c)$ that lies in the interior of one of the three discs, and then evaluate using (1),(2), and (4). Direct computations show that these two ways to evaluate a theta-foam give the same answer.

Rule (4) is related to the cohomology ring of the full flag variety $F_{3}$ of $\mathbb{C}^{3}$. The latter has degree 2 generators $X_{1}, X_{2}, X_{3}$ and defining relations

$$
\begin{aligned}
X_{1}+X_{2}+X_{3} & =0 \\
X_{1} X_{2}+X_{1} X_{3}+X_{2} X_{3} & =0 \\
X_{1} X_{2} X_{3} & =0 .
\end{aligned}
$$

The trace form

$$
\operatorname{Tr}: \mathrm{H}^{6}\left(F l_{3}, \mathbb{Z}\right) \longrightarrow \mathbb{Z}
$$

defined by $\operatorname{Tr}\left(X_{1} X_{2}^{2}\right)=1$ has the property

$$
\operatorname{Tr}\left(X_{1}^{a} X_{2}^{b} X_{3}^{c}\right)=\mathcal{F}(\theta(a, b, c)) .
$$

The dots on the discs of the theta foam correspond to generators $X_{1}, X_{2}, X_{3}$ of the flag variety cohomology ring.

Proposition 4 If a pre-foam $U_{1}$ is obtained from a pre-foam $U$ by reversing the cyclic order of the three annuli at a singular circle of $U$, then

$$
\mathcal{F}\left(U_{1}\right)=-\mathcal{F}(U)
$$

Define the Euler characteristic $\chi(U)$ of a pre-foam $U$ as the Euler characteristic of the two-dimensional $\mathrm{CW}$-complex underlying $U$ with all dots punctured out.

Proposition $5 \quad \mathcal{F}(U)=0$ if $\chi(U) \neq 0$.

Proposition $6 \mathcal{F}(U)=0$ in each of the following cases:

- a facet of $U$ has genus 2 or higher,

- a facet of $U$ has genus 1 and at least one dot,

- a facet of $U$ has at least 3 dots, 
- two facets of $U$ that share a singular circle have at least 4 dots,

- two annuli near a singular circle belong to the same facet of $U$.

\section{Pre-foam identities}

Rules (1)-(4) lead to several handy formulas for pre-foam evaluation, depicted in figures 17, 18, 19].
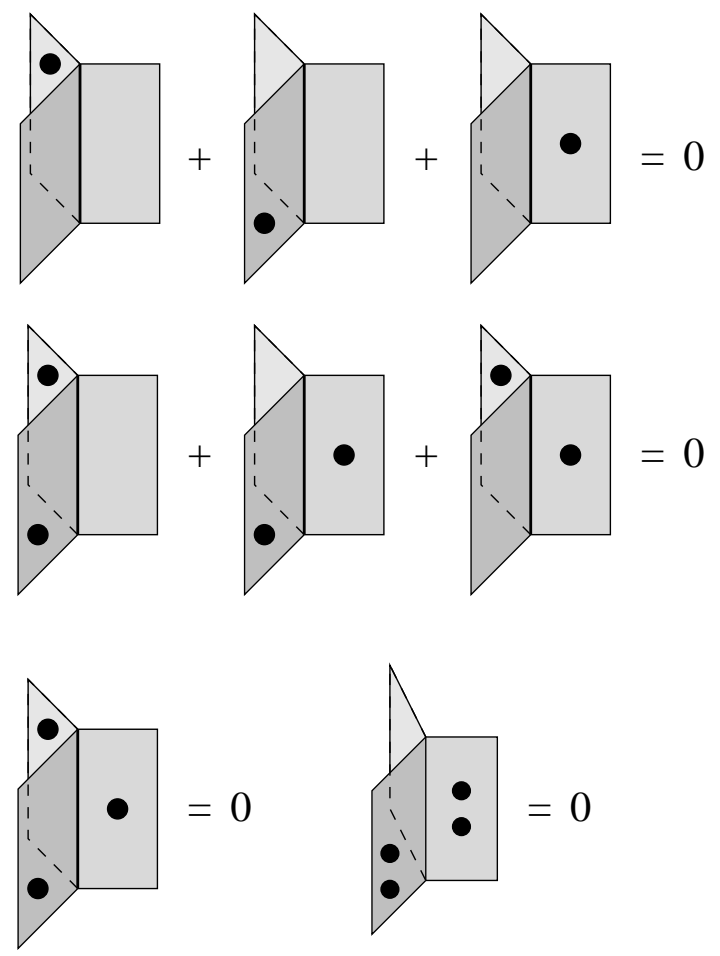

Figure 17: Relations involving dots and singular circles, compare with formulas (11)-(3)

\subsection{Foams and web homology}

Closed foams A closed foam is a pre-foam $U$ embedded in $\mathbb{R}^{3}$ with all facets oriented in such a way that the three annuli near each singular circle are compatibly oriented. Orientations of annuli induce orientations on singular circles. The orientation of a singular circle together with the standard orientation of $\mathbb{R}^{3}$ induces a cyclic ordering of the three annuli at this circle. We require this 

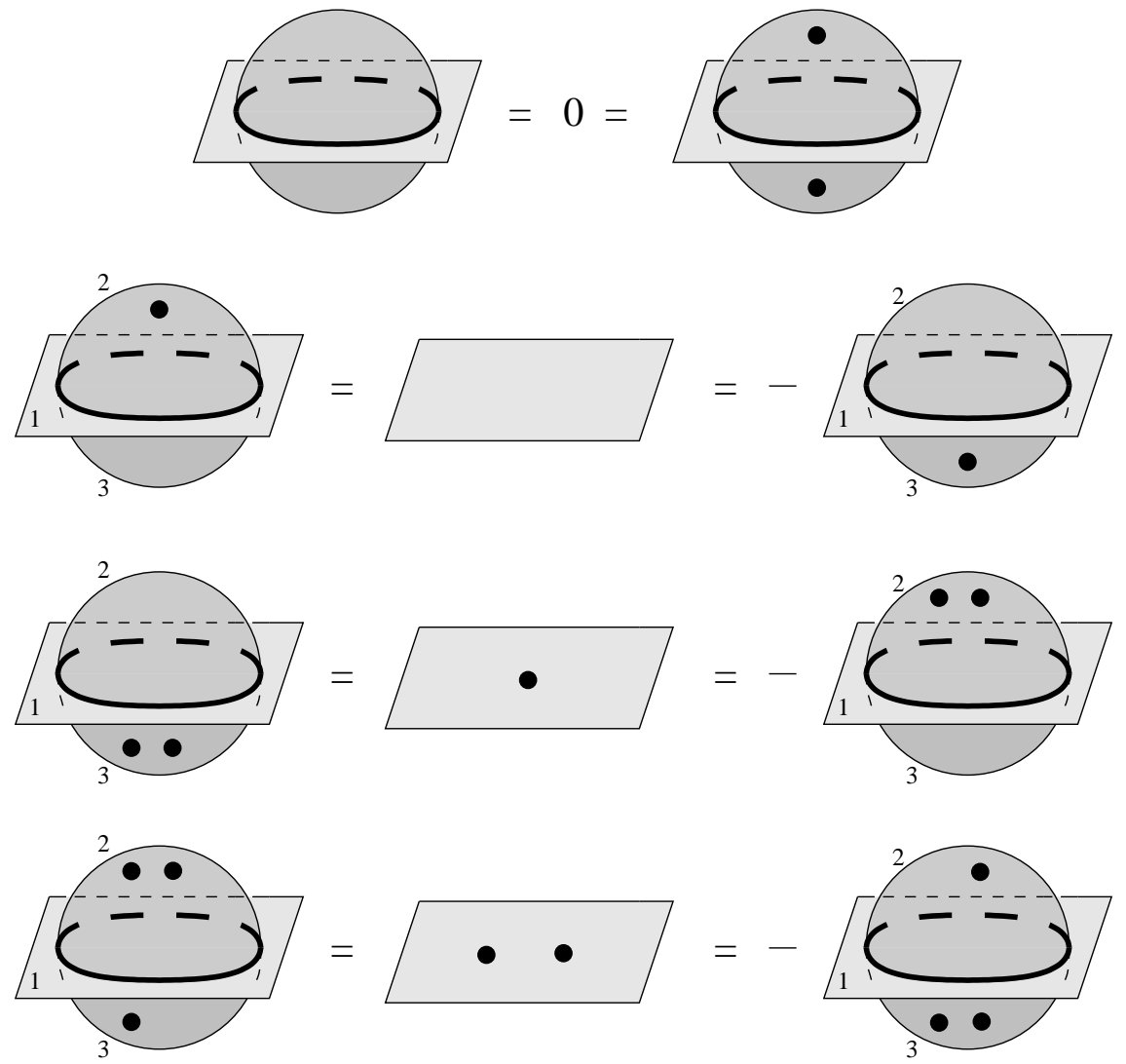

Figure 18: Bursting bubbles. Labels $1,2,3$ indicate the cyclic order of facets near a singular circle, $1 \rightarrow 2 \rightarrow 3 \rightarrow 1$

ordering to be the same as the ordering specified by the pre-foam $U$. Figure 20 explains our cyclic ordering convention.

There is an obvious forgetful map from closed foams to pre-foams. Define the evaluation $\mathcal{F}(U) \in \mathbb{Z}$ of a closed foam $U$ as the evaluation of the corresponding pre-foam.

Foams with boundary Let $T \cong \mathbb{R}^{2}$ be an oriented plane in $\mathbb{R}^{3}$. We say that $T$ intersects a closed foam $U$ generically if $T \cap U$ is a web in $T$. Note that the orientations of facets of $U$ induce orientations on edges of $T \cap U$.

Define a foam with boundary as the intersection of a closed foam $U$ and $T \times$ $[0,1] \subset \mathbb{R}^{3}$ such that $T \times\{0\}$ and $T \times\{1\}$ intersect $U$ generically. We think 

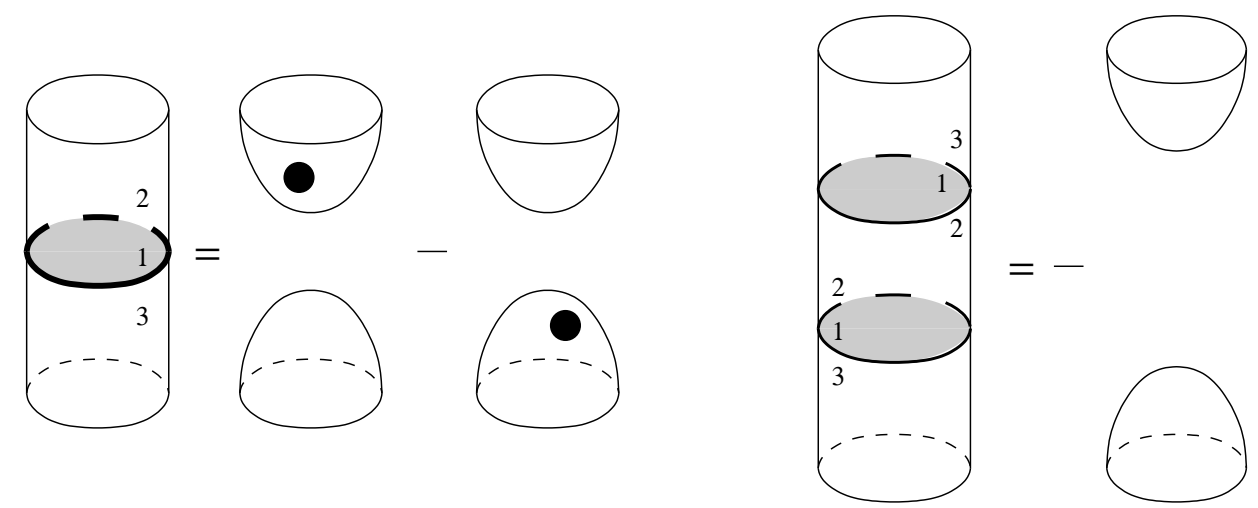

Figure 19: Removing discs

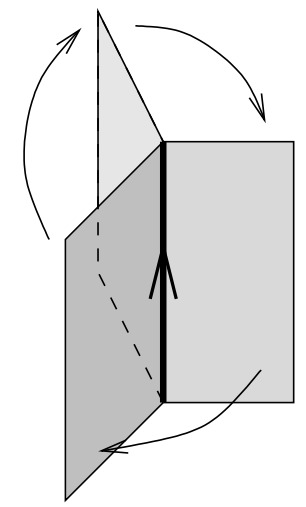

Figure 20: Cyclic ordering of annuli determined by singular circle orientation

of a foam $V$ with boundary as a cobordism between webs $\partial_{i} V \stackrel{\text { def }}{=} V \cap T(\times\{i\})$ for $i=0,1$. We will always consider the standard embedding of $T \times[0,1] \cong$ $\mathbb{R}^{2} \times[0,1]$ into $\mathbb{R}^{3}$. By a foam we will mean a foam with boundary. We say that two foams are isomorphic if they are isotopic in $\mathbb{R}^{2} \times[0,1]$ through an isotopy during which the boundary is fixed.

Example A closed foam is a foam with the empty boundary, and a cobordism between the empty webs.

If $U, V$ are foams and webs $\partial_{0} U, \partial_{1} V$ are identical as oriented graphs in $\mathbb{R}^{2}$ (not just isotopic, but isomorphic), we define the composition $U V$ in the obvious way. It is straightforward to check that any new singular circle that appears 
in the composition has a neighbourhood homeomorphic to $Y \times \mathbb{S}^{1}$, and not to a nontrivial $Y$-bundle over $\mathbb{S}^{1}$. Otherwise, the intersection of $U V$ with the boundary of a solid torus neighbourhood of that singular circle is a simple closed curve $t$ on the 2-torus $T^{2}$ wrapping three times around the longitude and once or twice along the meridian. Thus, the curve represents a nontrivial element in $H^{1}\left(T^{2}, \mathbb{Z}_{3}\right)$. On the other hand, the intersection of $U V$ with the complement of the same neighbourhood of the singular circle gives us a $\mathbb{Z}_{3}$ two-chain in the complement with $t$ as its boundary (to produce the two-chain, contract boundary graphs of $U V$ into points). Contradiction. Therefore, $U V$ is a foam.

Let Foams be the category with webs as objects and isomorphism classes of foams (rel boundary) as morphisms. A foam $U$ is a morphism from $\partial_{0} U$ to $\partial_{1} U$. Define the degree of $U$ as

$$
\operatorname{deg}(U) \stackrel{\text { def }}{=} \chi(\partial U)-2 \chi(U)
$$

where $\chi$ is the Euler characteristic. $\operatorname{deg}(U V)=\operatorname{deg}(U)+\operatorname{deg}(V)$ for any composable foams $U, V$.

We now define homology $\mathcal{F}(\Gamma)$ of a web $\Gamma$.

Definition $\mathcal{F}(\Gamma)$ is an abelian group generated by symbols $\mathcal{F}(U)$ for all foams $U$ from the empty web $\emptyset$ to $\Gamma$. A relation $\sum_{i} a_{i} \mathcal{F}\left(U_{i}\right)=0$ for $a_{i} \in \mathbb{Z}$ and $U_{i} \in \operatorname{Hom}_{\text {Foams }}(\emptyset, \Gamma)$ holds in $\mathcal{F}(\Gamma)$ if and only if

$$
\sum_{i} a_{i} \mathcal{F}\left(V U_{i}\right)=0
$$

for any foam $V$ from $\Gamma$ to the empty web, where $\mathcal{F}\left(V U_{i}\right) \in \mathbb{Z}$ is the evaluation of the closed foam $V U_{i}$.

Example The homology of the empty web is $\mathbb{Z}$.

Since $\operatorname{deg}\left(V U_{i}\right)=\operatorname{deg}(V)+\operatorname{deg}\left(U_{i}\right)$ and $\mathcal{F}\left(V U_{i}\right)=0$ if $\operatorname{deg}\left(V U_{i}\right)=-2 \chi\left(V U_{i}\right) \neq$ 0 , the group $\mathcal{F}(\Gamma)$ is naturally graded by $\operatorname{deg}(U)$.

To a foam $U$ there is associated a homomorphism of abelian groups

$$
\mathcal{F}(U): \mathcal{F}\left(\partial_{0} U\right) \longrightarrow \mathcal{F}\left(\partial_{1} U\right)
$$

that takes $\mathcal{F}(V)$, for any foam $V \in \operatorname{Hom}_{\text {Foams }}\left(\emptyset, \partial_{0} U\right)$, to $\mathcal{F}(U V)$. This homomorphism has degree $\operatorname{deg}(U)$.

We will often depict a dotless foam $U$ by a sequence of its cross-sections $U \cap$ $\left(R^{2} \times\{t\}\right)$ for several $t \in[0,1]$, starting with $t=0$ and ending with $t=1$. Such presentations for foams with only one non-generic cross-section $U \cap\left(R^{2} \times\right.$ $\{s\}), s \in[0,1]$ are given in Figure 21] with $t=0,1$ for each foam. We call these foams basic foams. 

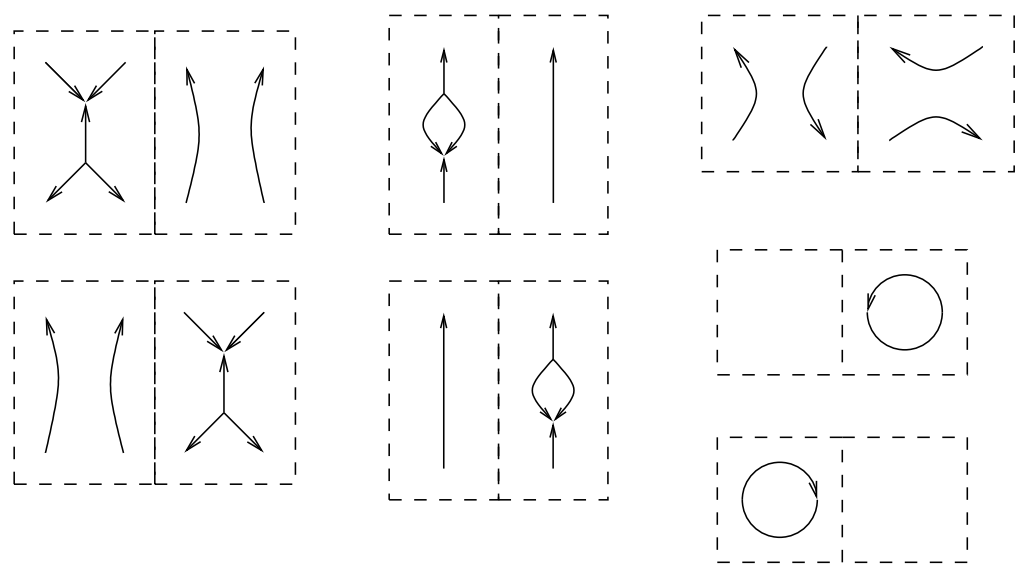

Figure 21: Basic foams

\subsection{Web homology skein relations}

Our goal in this section is to show that $\mathcal{F}(\Gamma)$ is a free graded abelian group of graded rank $\langle\Gamma\rangle$ that satisfies skein relations categorifying those of $\langle\Gamma\rangle$, the latter depicted in Figure 7 .

Compatibility A web $\Gamma$ without trivalent vertices is a closed oriented 1manifold embedded in $\mathbb{R}^{2}$. Let us show that the two definitions of $\mathcal{F}(\Gamma)$ for such $\Gamma$, one in Section 3.1 and the other in Section 3.3 give canonically isomorphic abelian groups. To temporarily distinguish them, we call the first $\mathcal{F}_{[3.1}(\Gamma)$ and the second $\mathcal{F}_{[3.3}(\Gamma)$.

Define the following maps:

$$
\mathcal{F}_{[3.1}(\Gamma) \stackrel{\alpha}{\longrightarrow} \mathcal{F}_{[3.3}(\Gamma), \quad \mathcal{F}_{[3.3}(\Gamma) \stackrel{\beta}{\longrightarrow} \mathcal{F}_{[3.1}(\Gamma) .
$$

$\mathcal{F} \mathcal{F B . 1}_{1}(\Gamma) \cong \mathcal{A}^{\otimes n}$, where $n$ is the number of connected components of $\Gamma$. This abelian group has a basis of elements $X^{a_{1}} \otimes X^{a_{2}} \otimes \cdots \otimes X^{a_{n}}$ for $a_{i} \in\{0,1,2\}$. We let $\alpha$ take this element to the foam from the empty web to $\Gamma$ which is a union of cups, one for each component of $\Gamma$, decorated by $a_{i}$ dots, see example in Figure 22

To define $\beta$, start with a foam $U$ from the empty web to $\Gamma$, and do a surgery near each boundary circle of $U$. Each term in the resulting sum is a disjoint union of a dotted cup foam and a closed foam. Evaluate closed foams using $\mathcal{F}$ and convert dotted cup foams to basis elements of $\mathcal{F}_{[3.1}(\Gamma)$ by inverting the 


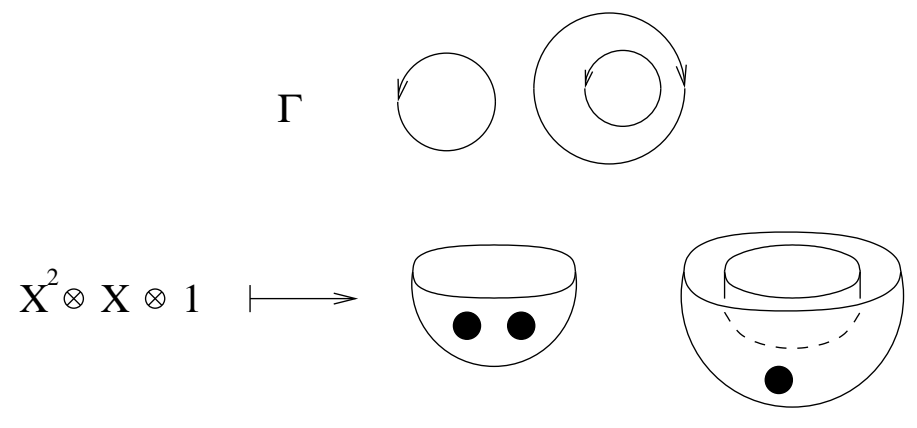

Figure 22: Map $\alpha$

procedure in Figure 22. The sum becomes an element of $\mathcal{F}_{[3.3}(\Gamma)$, and we define $\beta(U)$ as this sum. It is easy to see that $\beta$ is well-defined, and is a two-sided inverse of $\alpha$.

\section{Adding a circle}

Proposition 7 If $\Gamma$ is obtained from $\Gamma_{1}$ by adding an innermost circle $P$ then

$$
\mathcal{F}(\Gamma) \cong \mathcal{F}\left(\Gamma_{1}\right) \otimes \mathcal{F}(P) .
$$

Remark For an example of such $\Gamma$ see Figure 6. Note that $\mathcal{F}(P) \cong \mathcal{A}$.

Proof We leave the proof to the reader. It is similar to the above proof that the two definitions of $\mathcal{F}$ are compatible on verticeless $\Gamma$.

Removing a digon face Suppose that $\Gamma_{1}$ is given by removing a digon face from $\Gamma$, see Figure 23

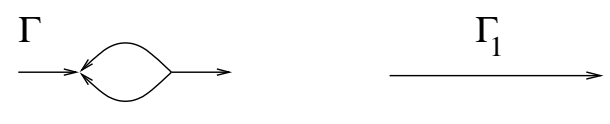

Figure 23: Digon removal

Proposition 8 There is a natural isomorphism of graded abelian groups

$$
\mathcal{F}(\Gamma) \cong \mathcal{F}\left(\Gamma_{1}\right)\{1\} \oplus \mathcal{F}\left(\Gamma_{1}\right)\{-1\} .
$$



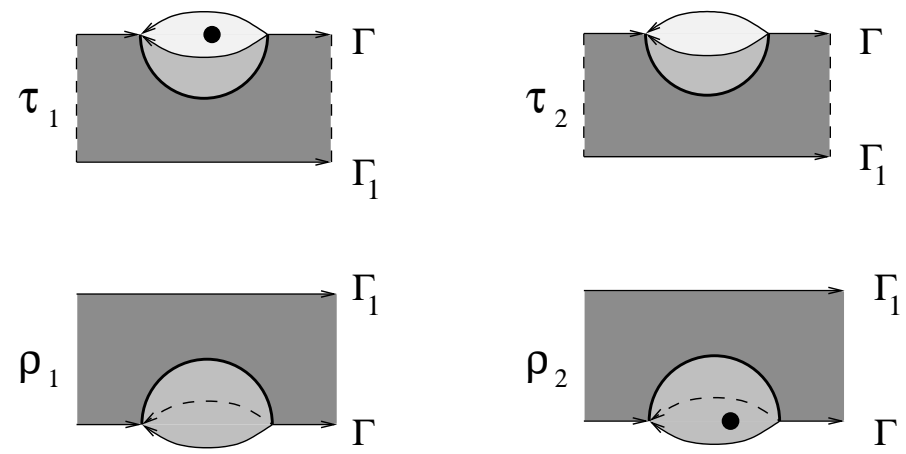

Figure 24: Web cobordisms $\tau_{1}, \tau_{2}, \rho_{1}, \rho_{2}$

Proof Consider the cobordisms $\rho_{1}, \rho_{2}, \tau_{1}, \tau_{2}$ between $\Gamma$ and $\Gamma_{1}$ depicted in Figure 24.

These cobordisms induce grading-preserving maps

$$
\begin{aligned}
\mathcal{F}\left(\Gamma_{1}\right)\{1\} \stackrel{\mathcal{F}\left(\tau_{1}\right)}{\longrightarrow} \mathcal{F}(\Gamma), & \mathcal{F}(\Gamma) \stackrel{\mathcal{F}\left(\rho_{1}\right)}{\longrightarrow} \mathcal{F}\left(\Gamma_{1}\right)\{1\}, \\
\mathcal{F}\left(\Gamma_{1}\right)\{-1\} \stackrel{\mathcal{F}\left(\tau_{2}\right)}{\longrightarrow} \mathcal{F}(\Gamma), & \mathcal{F}(\Gamma) \stackrel{\mathcal{F}\left(\rho_{2}\right)}{\longrightarrow} \mathcal{F}\left(\Gamma_{1}\right)\{-1\} .
\end{aligned}
$$

We claim that

$$
\begin{aligned}
\mathcal{F}\left(\rho_{1} \tau_{1}\right) & =\operatorname{Id}_{\Gamma_{1}} \\
-\mathcal{F}\left(\rho_{2} \tau_{2}\right) & =\operatorname{Id}_{\Gamma_{1}} \\
\mathcal{F}\left(\rho_{1} \tau_{2}\right) & =0 \\
\mathcal{F}\left(\rho_{2} \tau_{1}\right) & =0 \\
\mathcal{F}\left(\tau_{1} \rho_{1}\right)-\mathcal{F}\left(\tau_{2} \rho_{2}\right) & =\operatorname{Id}_{\Gamma} .
\end{aligned}
$$

Indeed, the first four equalities follow from identities for pre-foams, see figures 17, 18. The last identity is depicted in Figure 25]

To prove it we show that for any foams $U \in \operatorname{Hom}_{\text {Foams }}(\emptyset, \Gamma)$ and $V \in \operatorname{Hom}_{\text {Foams }}(\Gamma, \emptyset)$ there is an equality of closed foam evaluations

$$
\mathcal{F}(V U)=\mathcal{F}\left(V \tau_{1} \rho_{1} U\right)-\mathcal{F}\left(V \tau_{2} \rho_{2} U\right) .
$$

There are two cases to consider:

(1) the two singular intervals of $\operatorname{Id}_{\Gamma}$ shown in Figure 25] belong to the same singular circle of $V U \cong V \operatorname{Id}_{\Gamma} U$.

(2) these intervals belong to different singular circles of $V U$. 

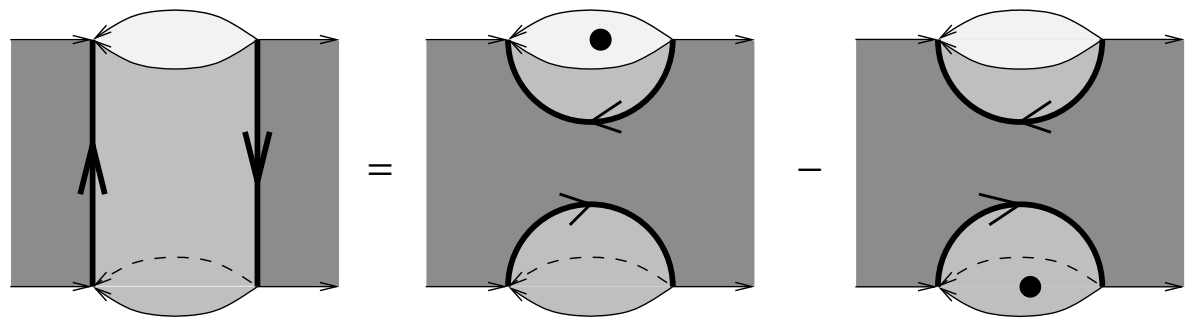

Figure 25: Identity of $\mathcal{F}(\Gamma)$ decomposed

In each case we do surgeries on $V U$ near all of its singular circles that do not intersect the piece of $V U$ shown in Figure 25 left, and separate these circles from that piece. Identical surgeries are done on the other two closed foams in (5). After this operation we are reduced to checking the validity of (5) in the following cases:

(1) $V U$ is a theta foam. Foams $V \tau_{1} \rho_{1} U$ and $V \tau_{2} \rho_{2} U$ then each have two singular circles.

(2) $V U$ has two singular circles and is a "connected sum" of two theta foams. $V \tau_{1} \rho_{1} U$ and $V \tau_{2} \rho_{2} U$ are theta foams.

These cases are verified by hand, using relations in figures [17, 18, 19,

The five equalities above imply that maps $\left(\mathcal{F}\left(\rho_{1}\right), \mathcal{F}\left(\rho_{2}\right)\right)$ and $\left(\mathcal{F}\left(\tau_{1}\right),-\mathcal{F}\left(\tau_{2}\right)\right)$ between the left and right hand sides of (4) are mutually inverse isomorphisms.

\section{Square face}

Proposition 9 If $\Gamma_{1}, \Gamma_{2}$ are two reductions of a web $\Gamma$ with a square face, see Figure 26, there is a canonical isomorphism

$$
\mathcal{F}(\Gamma) \cong \mathcal{F}\left(\Gamma_{1}\right) \oplus \mathcal{F}\left(\Gamma_{2}\right) .
$$

Let $\nu_{1}, \nu_{2}, \psi_{1}, \psi_{2}$ be the cobordisms between $\Gamma, \Gamma_{1}$, and $\Gamma_{2}$ depicted in Figure 27 and denoted by arrows in Figure 26.

We claim that

$$
\begin{aligned}
\mathcal{F}\left(\psi_{1} \nu_{1}\right) & =-\operatorname{Id}_{\Gamma_{1}} \\
\mathcal{F}\left(\psi_{2} \nu_{2}\right) & =-\operatorname{Id}_{\Gamma_{2}} \\
\mathcal{F}\left(\psi_{2} \nu_{1}\right) & =0 \\
\mathcal{F}\left(\psi_{1} \nu_{2}\right) & =0 \\
\mathcal{F}\left(\nu_{1} \psi_{1}\right)+\mathcal{F}\left(\nu_{2} \psi_{2}\right) & =-\operatorname{Id}_{\Gamma} .
\end{aligned}
$$




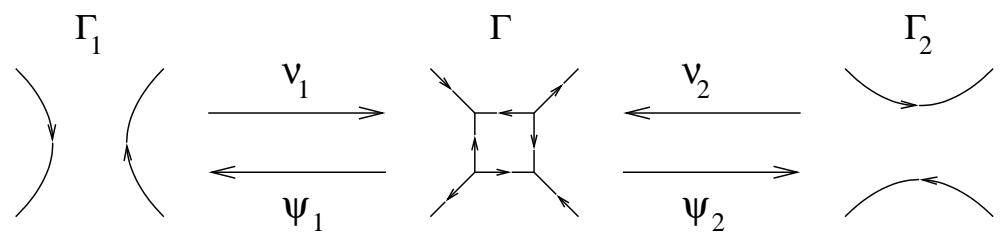

Figure 26: Scheme of cobordisms between $\Gamma$ and its reductions
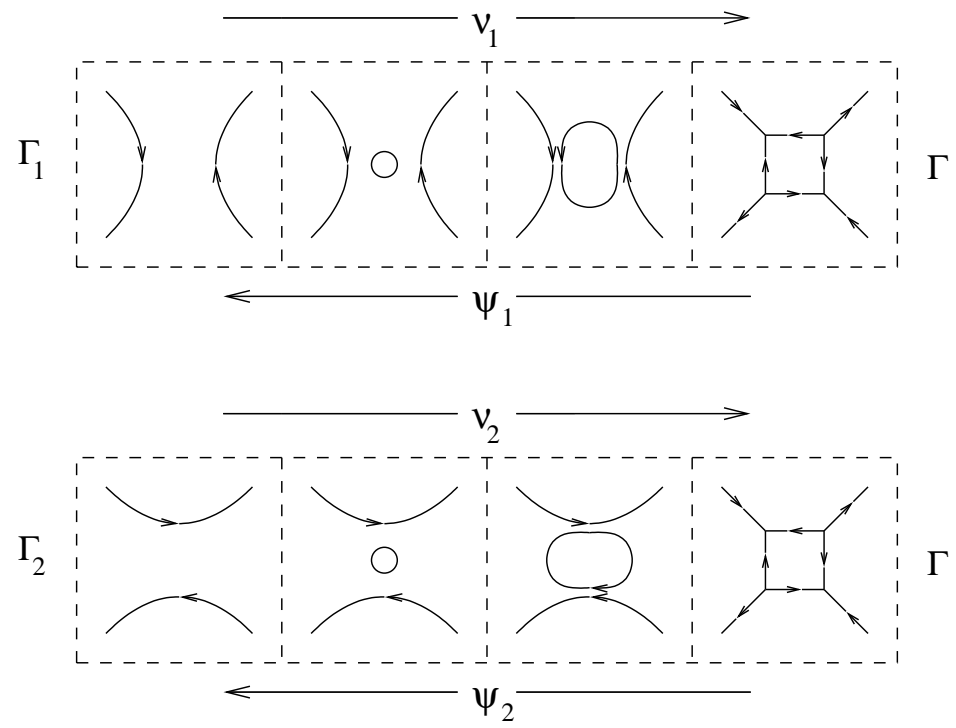

Figure 27: Cobordisms $\nu_{1}, \nu_{2}, \psi_{1}, \psi_{2}$

Indeed, (7) and (8) are equivalent to the equality in Figure 19 right, and (9) and (10) are equivalent to the top left equality in Figure 18.

To prove the last equality, rewrite it as

$$
\mathcal{F}\left(\nu_{1} \psi_{1}\right)+\mathcal{F}\left(\nu_{2} \psi_{2}\right)+\operatorname{Id}_{\Gamma}=0 .
$$

We need to show that, for any $V \in \operatorname{Hom}_{\text {Foams }}(\emptyset, \Gamma)$ and $U \in \operatorname{Hom}_{\text {Foams }}(\Gamma, \emptyset)$, the following relation between three integers holds:

$$
\mathcal{F}\left(U \nu_{1} \psi_{1} V\right)+\mathcal{F}\left(U \nu_{2} \psi_{2} V\right)+\mathcal{F}(U V)=0 .
$$

Non-trivial parts of web cobordisms $\nu_{1} \psi_{1}$ and $\nu_{2} \psi_{2}$ lie in $D^{2} \times[0,1]$, the direct product of a 2-disc and the interval $[0,1]$. Smooth out the sharp edges of $D^{2} \times$ $[0,1]$ slightly so that its boundary is a 2 -sphere in $\mathbb{R}^{3}$. Denote this sphere by $S$ 
and the 3 -disc that it bounds by $D^{3}$. Each of the cobordisms $\nu_{1} \psi_{1}, \nu_{2} \psi_{2}$, and $\operatorname{Id}_{\Gamma}$ intersect $S$ along the same web $\Sigma$, isomorphic to the cube web, Figure 28

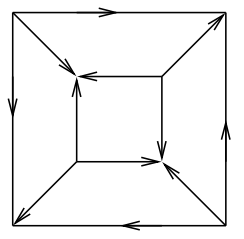

Figure 28: Cube web $\Sigma$

Each of the three cobordisms $U \nu_{1} \psi_{1} V, U \nu_{2} \psi_{2} V$ and $U V \cong U \operatorname{Id}_{\Gamma} V$ has identical intersection, denoted $E$, with the complement of $D^{3}$ in $\mathbb{R}^{3}$. This intersection can be viewed as a foam whose boundary is $\Sigma$. Apply a diffeomorphism of $\mathbb{S}^{3}=\mathbb{R}^{3} \cup\{\infty\}$ that takes $S$ to $\mathbb{R}^{2} \cup\{\infty\}$. This diffeomorphism takes $\Sigma$ to a planar web, also denoted $\Sigma$, and $E$ to a foam in $\mathbb{R}^{3}$ with boundary $\Sigma$. The images $\mu_{1}, \mu_{2}, \mu_{3}$ of intersections of $U \nu_{1} \psi_{1} V, U \nu_{2} \psi_{2} V$ and $U V$ with $D^{3}$ are foams with boundary $\Sigma$, depicted in Figure 29.
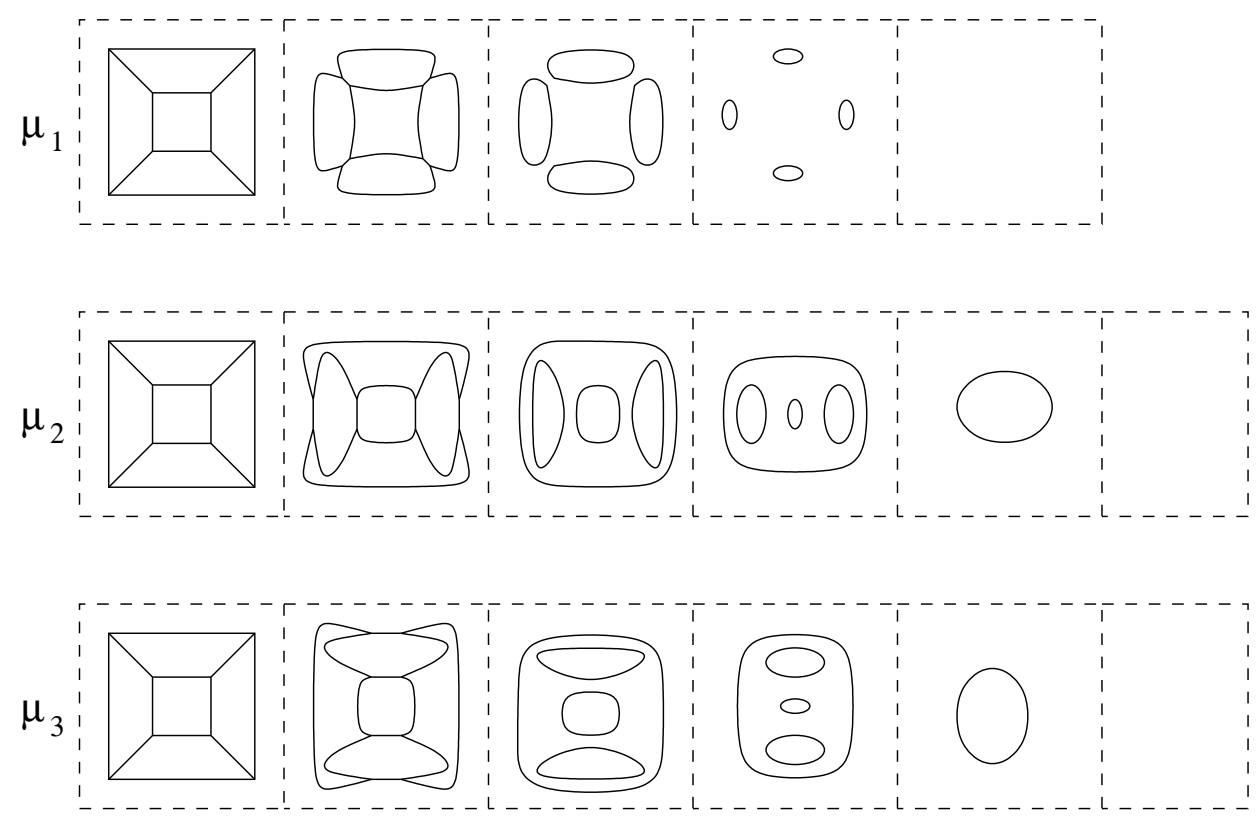

Figure 29: Cobordisms $\mu_{1}, \mu_{2}, \mu_{3}$ from $\Sigma$ to the empty web. Orientations of edges are omitted to avoid clutter. 
This transformation preserves the values of all closed foams. Therefore, it suffices to show that

$$
\mathcal{F}\left(\mu_{1} E\right)+\mathcal{F}\left(\mu_{2} E\right)+\mathcal{F}\left(\mu_{3} E\right)=0
$$

for any foam $E \in \operatorname{Hom}_{\text {Foams }}(\emptyset, \Sigma)$. The foam $E$ contains four singular arcs that pair up the eight vertices of $\Sigma$, each arc connecting an "in" vertex with an "out" vertex.

Suppose that one of these arcs connects two neighbouring vertices $v_{1}, v_{2}$ of $\Sigma$. One of the three foams $\mu_{1}, \mu_{2}, \mu_{3}$ contains a singular arc connecting the same pair of vertices. Since $\Sigma$ has automorphisms that extend to cyclic permutations of $\mu_{1}, \mu_{2}, \mu_{3}$, we can assume that this foam is $\mu_{1}$. Simplify closed foams $\mu_{1} E, \mu_{2} E, \mu_{3} E$ by cutting off singular circles that are disjoint from $\Sigma$. The foam $\mu_{1} E$ has a facet containing the edge of $\Sigma$ that joins vertices $v_{1}$ and $v_{2}$. Using relations in figures 14 and [17 reduce the genus of this facet to 0 and move all dots away from it to other parts of $E$. Now apply the relation in Figure 19 left to the resulting foam, and the Figure 25] equality at certain digon sections of $\mu_{2} E$ and $\mu_{3} E$. Everything in the left hand side of (12) cancels and identity (12) follows in this case.

The only other case to consider is when each of the four arcs of $E$ connect a vertex of $\Sigma$ with the opposite vertex (the one that is three edges away). To treat this case, do surgery on $E$ to remove singular circles that do not intersect $\Sigma$ and reduce the genus of all facets of $E$ to 0 . The Euler characteristic of $\mu_{i} E$ is then $-4+2 m$ where $m$ is the number of dots on (modified) $E$. Unless $E$ has exactly two dots, pre-foams $\mu_{1} E, \mu_{2} E, \mu_{3} E$ have non-zero Euler characteristic and their evaluations equal 0 . Furthermore, ignoring the dots, pre-foams $\mu_{1} E, \mu_{2} E, \mu_{3} E$ are isomorphic (these isomorphisms come from automorphisms of $\Sigma$ that extend to modified $E$, the latter viewed as a pre-foam with boundary). Move the two dots close to each other and to a vertex of $\Sigma$. Relation (12) now follows from the top two relations in Figure 17.

We have shown that

$$
\mathcal{F}\left(\mu_{1}\right)+\mathcal{F}\left(\mu_{2}\right)+\mathcal{F}\left(\mu_{3}\right)=0,
$$

which is equivalent to (11).

Equations (17) through (11) imply that $\left(\mathcal{F}\left(\psi_{1}\right), \mathcal{F}\left(\psi_{2}\right)\right)$ and $\left(-\mathcal{F}\left(\nu_{1}\right),-\mathcal{F}\left(\nu_{2}\right)\right)$ are mutually inverse isomorphisms between the L.H.S. and the R.H.S. of (6) . Proposition 9 follows.

Corollary $2 \mathcal{F}(\Gamma)$ is a free abelian group of graded rank $\langle\Gamma\rangle$. 
Corollary 3 There is a natural isomorphism

$$
\mathcal{F}\left(\Gamma_{1} \sqcup \Gamma_{2}\right) \cong \mathcal{F}\left(\Gamma_{1}\right) \otimes \mathcal{F}\left(\Gamma_{2}\right),
$$

where $\Gamma_{1} \sqcup \Gamma_{2}$ is the disjoint union of webs $\Gamma_{1}, \Gamma_{2}$.

\section{Chain complex of a link diagram}

Given a plane diagram $D$ of a link $L$, let $I$ be the set of crossings of $D$, and $p_{+}$, respectively $p_{-}$, be the number of positive, respectively negative, crossings; $p_{+}+p_{-}=|I|$.

To $D$ we associate an $|I|$-dimensional cube of graded abelian groups. Vertices of the cube are in a bijection with subsets of $I$. To $J \subset I$ we associate a web $D_{J}$, which is the $J$-flattening of $D$. If $a \in J$, the crossing $a$ gets 1-flattening, otherwise it gets 0 -flattening, see Figure 9. In the vertex $J$ of the cube we place the graded abelian group $\mathcal{F}\left(D_{J}\right)\left\{3 p_{-}-2 p_{+}-|J|\right\}$, and to an inclusion $J \subset J b$, where $b \in I \backslash J$, and $J b$ denotes the set $J \sqcup\{b\}$, we assign the homomorphism

$$
\mathcal{F}\left(D_{J}\right)\left\{3 p_{-}-2 p_{+}-|J|\right\} \longrightarrow \mathcal{F}\left(D_{J b}\right)\left\{3 p_{-}-2 p_{+}-|J|-1\right\}
$$

induced by the basic cobordism between webs $D_{J}$ and $D_{J b}$, which looks like the cobordism in Figure 2 (if crossing $b$ is negative) or its reflection in a horizontal plane (if crossing $b$ is positive). Each of these homomorphisms is gradingpreserving. Functoriality of $\mathcal{F}$ implies that the cube is commutative.

We add minus signs to some maps to make each square in the cube anticommute. An intrinsic way to assign minus signs can be found, for example, in [6, Section 3.3]. Next form the total complex of this anticommutative cube, placing the first non-zero term, $\mathcal{F}\left(D_{\emptyset}\right)\left\{3 p_{-}-2 p_{+}\right\}$, in cohomological degree $-p_{-}$. Denote the total complex by $\mathcal{F}(D)$. It is non-zero in cohomological degrees between $-p_{-}$and $p_{+}$. The term of degree $-p_{-}+i$ is the following direct sum:

$$
\mathcal{F}(D)^{-p_{-}+i}=\underset{J \subset I,|J|=i}{\bigoplus} \mathcal{F}\left(D_{J}\right)\left\{3 p_{-}-2 p_{+}-i\right\} .
$$

The differential in the complex preserves the grading.

Figure 30 explains our construction of $\mathcal{F}(D)$. Corollary 2 implies that the Euler characteristic of $\mathcal{F}(D)$ is the Kuperberg bracket $\langle L\rangle$. In the next section we show that $\mathcal{F}(D)$, up to chain homotopy equivalence, is a link invariant. 


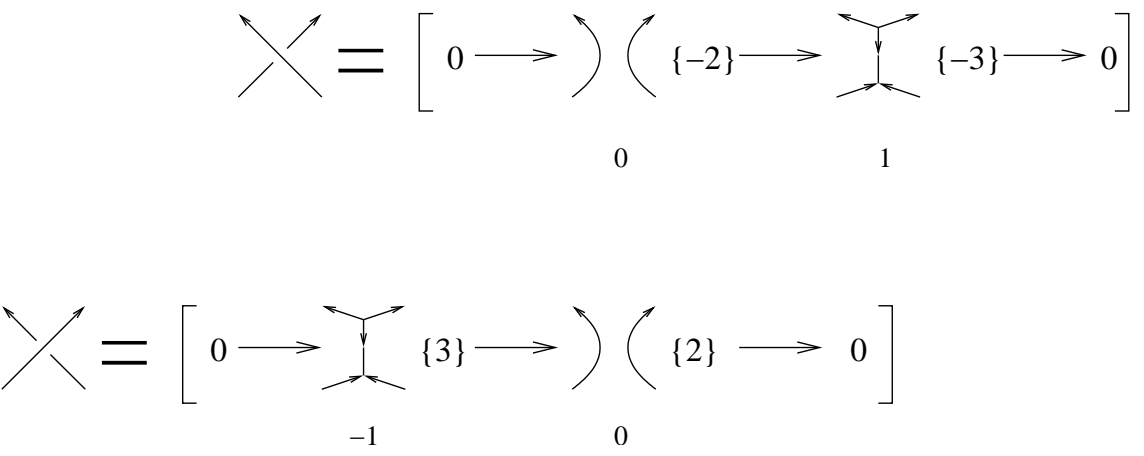

Figure 30: $-1,0,1$ underlining the flattenings indicate cohomological degree

\section{$5 \quad$ Invariance under Reidemeister moves}

Proofs of all propositions stated in this section are straightforward and left to the reader.

\section{$5.1 \quad$ Type I moves}

We kick-off by showing that complexes $\mathcal{F}(D)$ and $\mathcal{F}\left(D^{\prime}\right)$ are homotopy equivalent, for diagrams $D$ and $D^{\prime}$ depicted in Figure 31

D
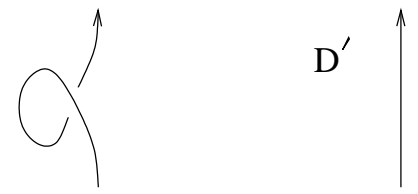

Figure 31: A type I move

Denote the flattenings of $D$ by $D_{0}$ and $D_{1}$. The complex $\mathcal{F}(D)$ is the total complex of the bicomplex

$$
0 \longrightarrow \mathcal{F}\left(D_{0}\right)\{-2\} \stackrel{h}{\longrightarrow} \mathcal{F}\left(D_{1}\right)\{-3\} \longrightarrow 0,
$$

see the top of Figure 32. The map $h$ is induced by the basic cobordism from $D_{0}$ to $D_{1}$.

Define $f: \mathcal{F}\left(D^{\prime}\right) \longrightarrow \mathcal{A} \otimes \mathcal{F}\left(D^{\prime}\right) \cong \mathcal{F}\left(D_{0}\right)$ by

$$
f(a)=X^{2} \otimes a+X \otimes X a+1 \otimes X^{2} a .
$$




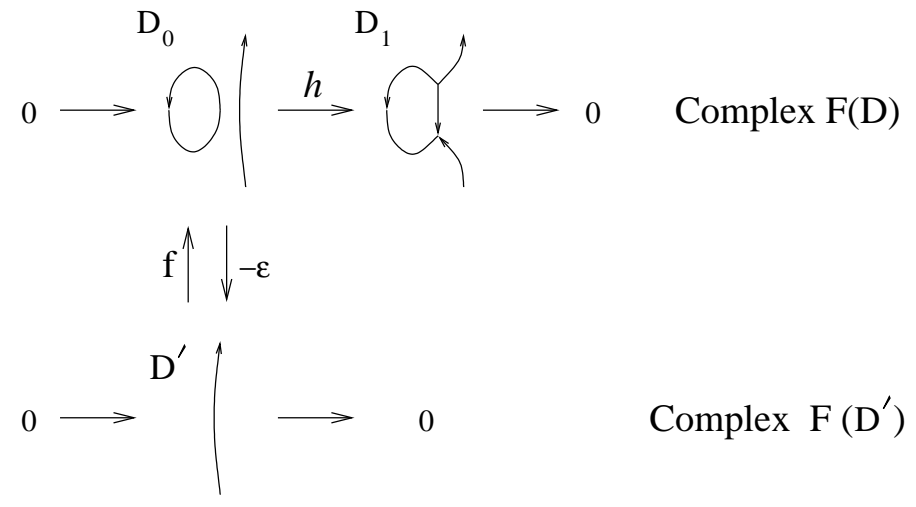

Figure 32: Realization of $\mathcal{F}\left(D^{\prime}\right)$ as a direct summand of $\mathcal{F}(D)$

Let $\varepsilon: \mathcal{F}\left(D_{0}\right) \rightarrow \mathcal{F}\left(D^{\prime}\right)$ be the trace map,

$$
\varepsilon(x \otimes a)=\varepsilon(x) a, \text { for } x \otimes a \in \mathcal{A} \otimes \mathcal{F}\left(D^{\prime}\right) \cong \mathcal{F}\left(D_{0}\right) .
$$

From the formulas $-\varepsilon f=\operatorname{Id}$ and $\operatorname{im}(f)=\operatorname{ker}(h)$ we get a direct sum decomposition

$$
\mathcal{F}(D) \cong f\left(\mathcal{F}\left(D^{\prime}\right)\right) \oplus\left\{0 \rightarrow \operatorname{ker}(\varepsilon) \rightarrow \mathcal{F}\left(D_{1}\right) \rightarrow 0\right\}
$$

The complex $f\left(\mathcal{F}\left(D^{\prime}\right)\right)$ is isomorphic to $\mathcal{F}\left(D^{\prime}\right)$, the second direct summand is contractible, since $h$, restricted to the kernel of $\varepsilon$, is an isomorphism of complexes. Therefore, $\mathcal{F}(D)$ and $\mathcal{F}\left(D^{\prime}\right)$ are homotopy equivalent.

We next take care of the other Reidemeister type I move, see Figure 33 .
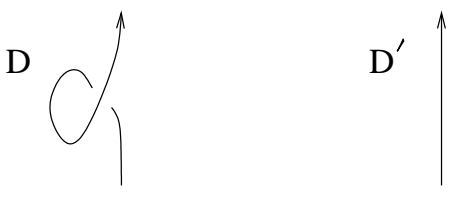

Figure 33: The other type I move

The complex $\mathcal{F}(D)$ is the total complex of the bicomplex

$$
0 \longrightarrow \mathcal{F}\left(D_{0}\right)\{3\} \stackrel{h}{\longrightarrow} \mathcal{F}\left(D_{1}\right)\{2\} \longrightarrow 0,
$$

see the top of Figure 34

Define $g: \mathcal{F}\left(D_{1}\right) \cong \mathcal{A} \otimes \mathcal{F}\left(D^{\prime}\right) \longrightarrow \mathcal{F}\left(D^{\prime}\right)$ by

$$
g(x \otimes a)=\varepsilon(x) X^{2} a+\varepsilon(X x) X a+\varepsilon\left(X^{2} x\right) a, \quad x \otimes a \in \mathcal{A} \otimes \mathcal{F}\left(D^{\prime}\right),
$$




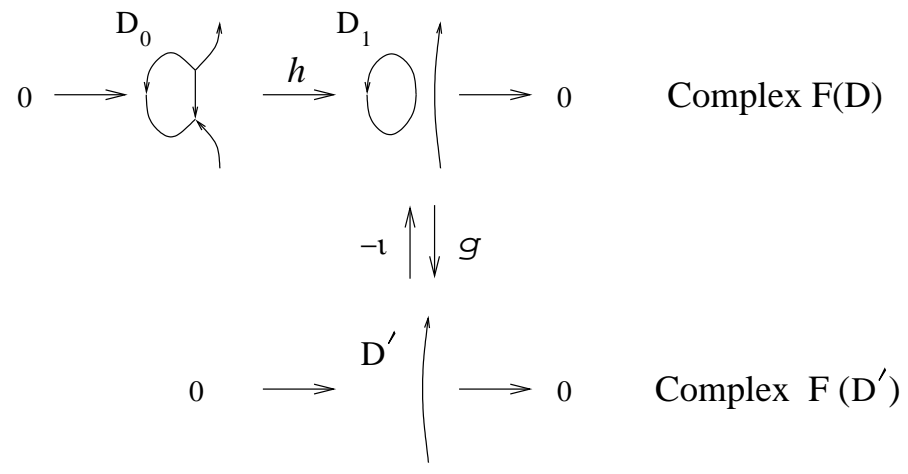

Figure 34: $\mathcal{F}\left(D^{\prime}\right)$ as a direct summand of $\mathcal{F}(D)$

and $\iota: \mathcal{F}\left(D^{\prime}\right) \longrightarrow \mathcal{A} \otimes \mathcal{F}\left(D^{\prime}\right) \cong \mathcal{F}\left(D_{1}\right)$ is the unit map, $\iota(a)=1 \otimes a$.

Since $-g \iota=\mathrm{Id}, g h=0$ and $\mathcal{F}\left(D_{1}\right) \cong h\left(\mathcal{F}\left(D_{0}\right)\right) \oplus \iota\left(\mathcal{F}\left(D^{\prime}\right)\right)$, there is a direct sum decomposition

$$
\mathcal{F}(D) \cong \iota\left(\mathcal{F}\left(D^{\prime}\right)\right) \oplus\left\{0 \rightarrow \mathcal{F}\left(D_{0}\right) \stackrel{h}{\rightarrow} \operatorname{ker}(g) \rightarrow 0\right\} .
$$

Complex $\iota\left(\mathcal{F}\left(D^{\prime}\right)\right)$ is isomorphic to $\mathcal{F}\left(D^{\prime}\right)$, while the second direct summand is contractible, since $h$ is an isomorphism onto $\operatorname{ker}(g)$. This establishes a homotopy equivalence between $\mathcal{F}(D)$ and $\mathcal{F}\left(D^{\prime}\right)$.

\section{$5.2 \quad$ Type II moves}
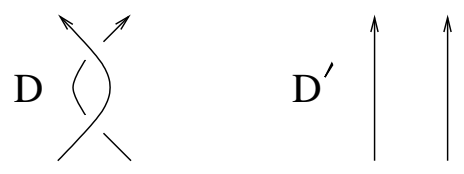

Figure 35: A type II move

Consider diagrams $D$ and $D^{\prime}$ in Figure 35. Figure 36 depicts the four flattenings of $D$. Arrows correspond to basic cobordisms, and a bar over the bottom arrow indicates that the map induced by this cobordism will be taken with the minus sign when the total complex of the commutative square in Figure 36 is formed.

Let $\alpha$ be the cobordism depicted in Figure 37 and $\beta$ the cobordism in Figure 38

Let

$$
Y_{1}=\left\{(x, \mathcal{F}(\alpha) x) \subset \mathcal{F}\left(D_{10}\right) \oplus \mathcal{F}\left(D_{01}\right), \quad x \in \mathcal{F}\left(D_{10}\right)\right\}
$$




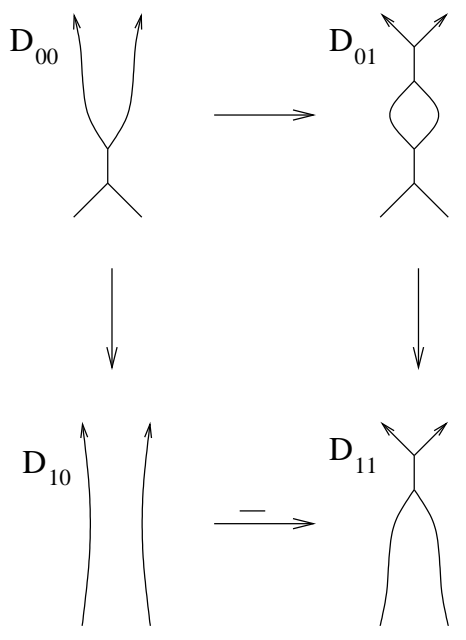

Figure 36: Flattenings of D

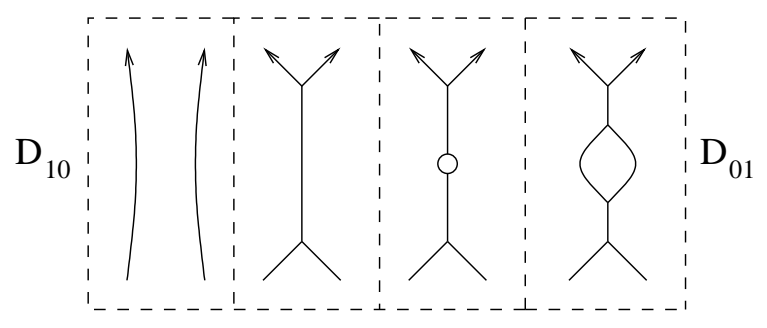

Figure 37: Cobordism $\alpha$

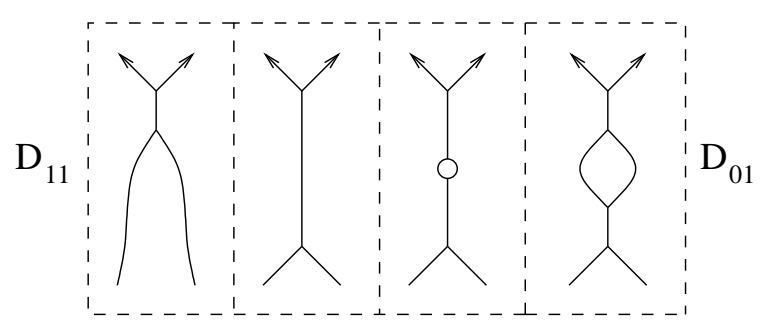

Figure 38: Cobordism $\beta$

Let $Y_{2}$ be the subcomplex of $\mathcal{F}(D)$ generated by $\mathcal{F}\left(D_{00}\right)$. Let

$$
Y_{3}=\left\{(\mathcal{F}(\beta) x, y) \subset \mathcal{F}\left(D_{01}\right) \oplus \mathcal{F}\left(D_{11}\right), \quad x, y \in \mathcal{F}\left(D_{11}\right)\right\}
$$

$Y_{3}$ is the subcomplex of $\mathcal{F}(D)$ generated by $\mathcal{F}(\beta) x$, over all $x \in \mathcal{F}\left(D_{11}\right)$. 
Proposition 10 (1) The complexes $Y_{2}$ and $Y_{3}$ are contractible.

(2) $Y_{1}$ is a subcomplex of $\mathcal{F}(D)$ isomorphic to $\mathcal{F}\left(D^{\prime}\right)$.

(3) $\mathcal{F}(D) \cong Y_{1} \oplus Y_{2} \oplus Y_{3}$.

Therefore, $\mathcal{F}(D)$ and $\mathcal{F}\left(D^{\prime}\right)$ are homotopy equivalent.

We next consider a type II move with a different orientation, see Figure 39
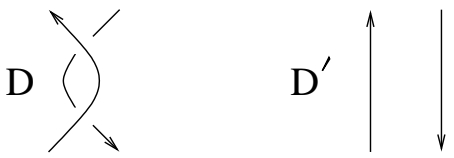

Figure 39: Another type II move

The four flattenings of $D$ are depicted in Figure 40.

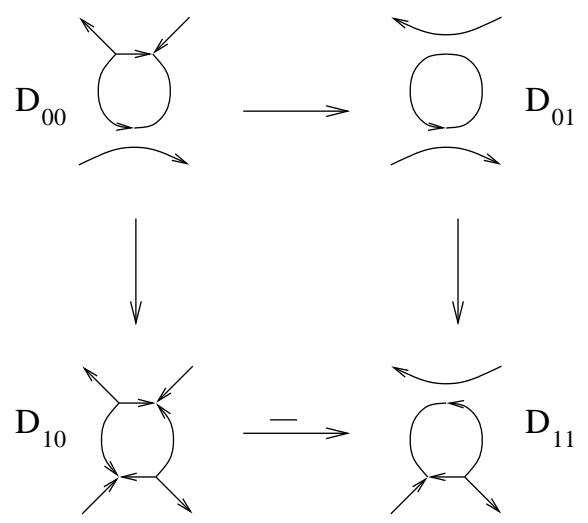

Figure 40: Flattenings of $D$

Let

$$
Y_{1}=\left(\mathcal{F}\left(\alpha_{1}\right) x, \mathcal{F}\left(\alpha_{2}\right) x\right) \subset \mathcal{F}\left(D_{10}\right) \oplus \mathcal{F}\left(D_{01}\right),
$$

where $x \in \mathcal{F}\left(D^{\prime}\right)$ and $\alpha_{1}, \alpha_{2}$ are Figure 41 cobordisms.

Let $Y_{2}$ be the subcomplex of $\mathcal{F}(D)$ generated by $\mathcal{F}\left(D_{00}\right)$.

The diagram $D_{01}$ contains a circle, see Figure 40 top right. Let $D^{\prime \prime}$ be the diagram given by removing this circle from $D_{01}$. Then $\mathcal{F}\left(D_{01}\right) \cong \mathcal{A} \otimes \mathcal{F}\left(D^{\prime \prime}\right)$. Let $Y_{3}$ be the subcomplex of $\mathcal{F}(D)$ generated by $1 \otimes \mathcal{F}\left(D^{\prime \prime}\right) \subset \mathcal{F}\left(D_{01}\right) \subset \mathcal{F}(D)$ and $X \otimes \mathcal{F}\left(D^{\prime \prime}\right) \subset \mathcal{F}\left(D_{01}\right) \subset \mathcal{F}(D)$. 

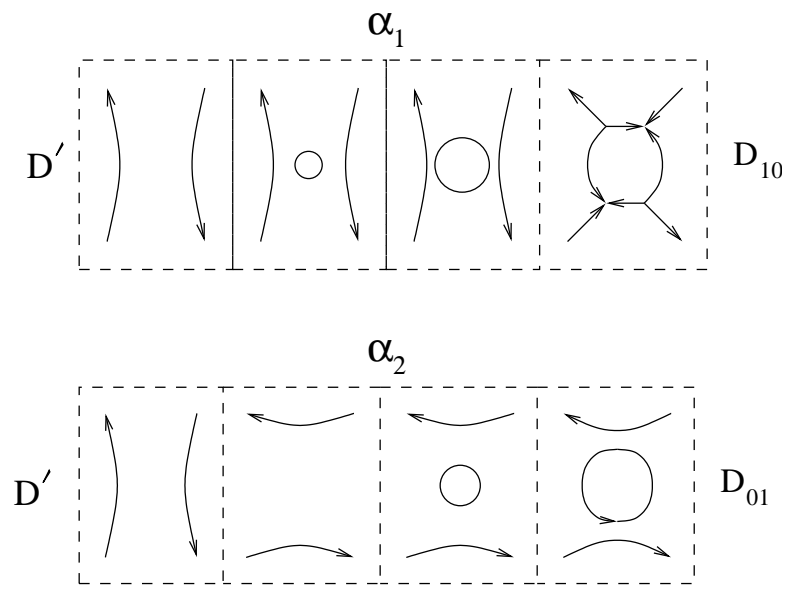

Figure 41: Cobordisms $\alpha_{1}$ and $\alpha_{2}$

Proposition 11 (1) Complexes $Y_{2}$ and $Y_{3}$ are contractible.

(2) $Y_{1}$ is a subcomplex of $\mathcal{F}(D)$ isomorphic to $\mathcal{F}\left(D^{\prime}\right)$.

(3) $\mathcal{F}(D) \cong Y_{1} \oplus Y_{2} \oplus Y_{3}$.

The proposition implies that $\mathcal{F}(D)$ and $\mathcal{F}\left(D^{\prime}\right)$ are homotopy equivalent.

\subsection{Type III move}

Any two moves of type III are equivalent modulo type I and II moves. Therefore, it suffices to treat only one case of the type III moves. We choose the one in Figure 42

\section{$\mathrm{D}$}
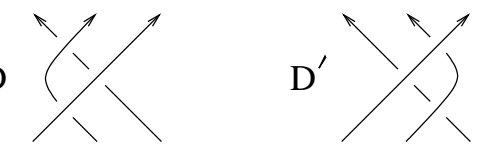

Figure 42: Reidemeister III move

$\mathcal{F}(D)$ is the total complex of the cube of 8 complexes shown in Figure 43 ,

Let $Q$ be the complex associated to Figure 44 It is the total complex of the bicomplex built out of complexes assigned to the six diagrams in Figure 44. Five 


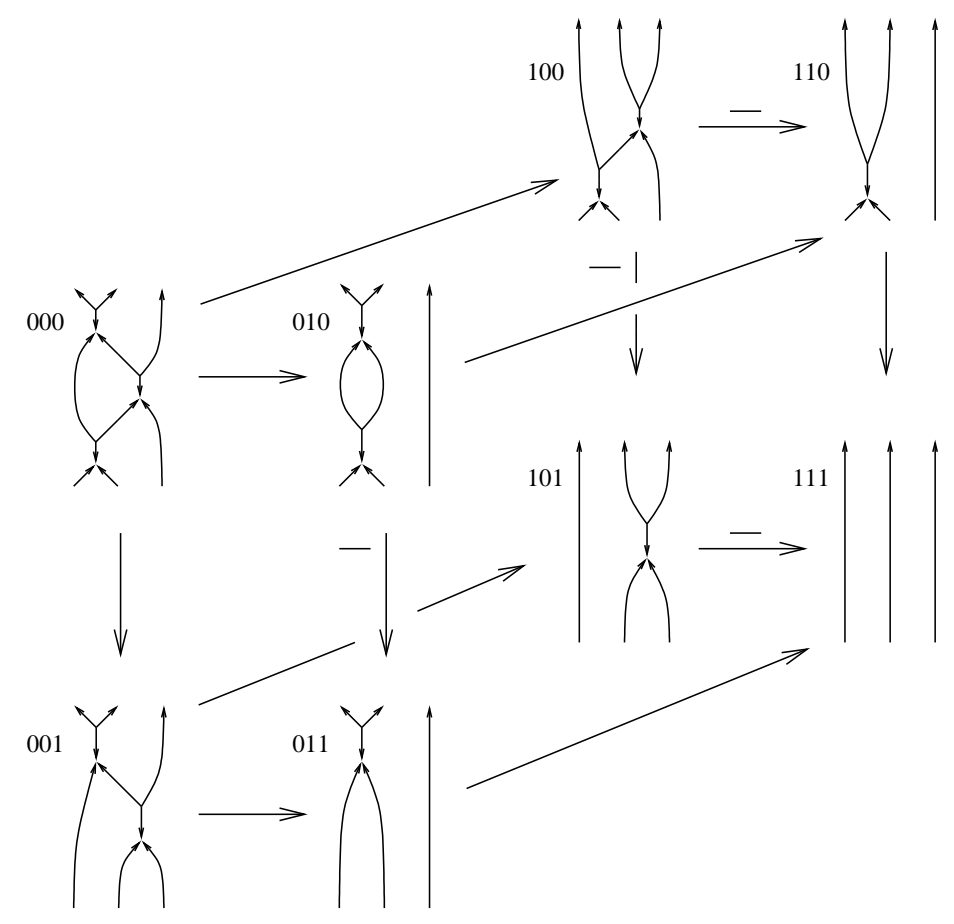

Figure 43: The cube of eight flattenings of $D$

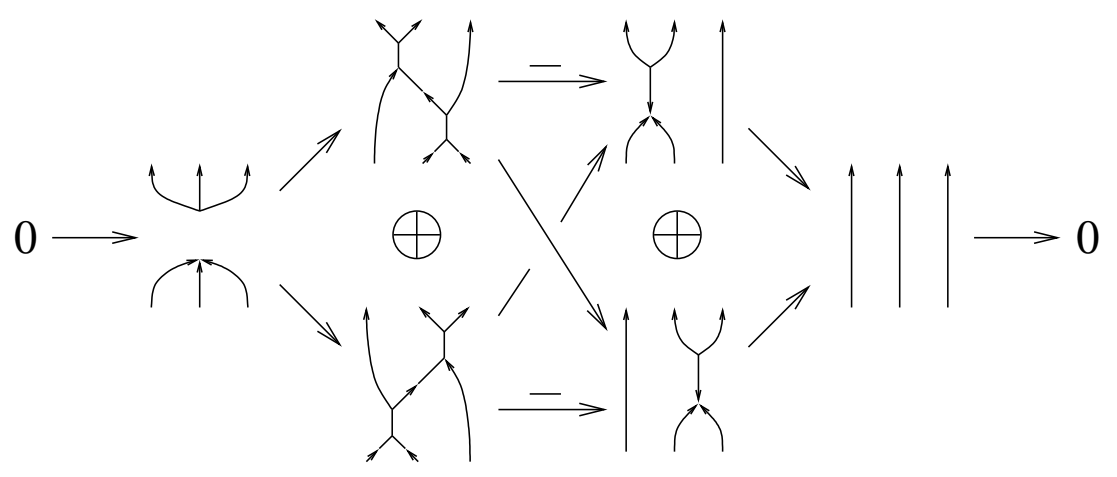

Figure 44: Complex $Q$

of these diagrams are $D_{001}, D_{100}, D_{101}, D_{110}, D_{111}$. The maps are induced by basic cobordisms between the diagrams.

Let $Y_{1}$ be the subcomplex of $\mathcal{F}(D)$ which, as an abelian group, is the direct 
sum of

- $\mathcal{F}\left(D_{1 i j}\right)$, over $i, j \in\{0,1\}$,

- $\mathcal{F}(W) \subset \mathcal{F}\left(D_{000}\right)$ where $W$ is the leftmost diagram in Figure 44 and the inclusion is induced by cobordism $\alpha$ in Figure 45 .

- $(x, \mathcal{F}(\beta) x)$, for $x \in \mathcal{F}\left(D_{001}\right)$ and cobordism $\beta$ depicted in Figure 45,
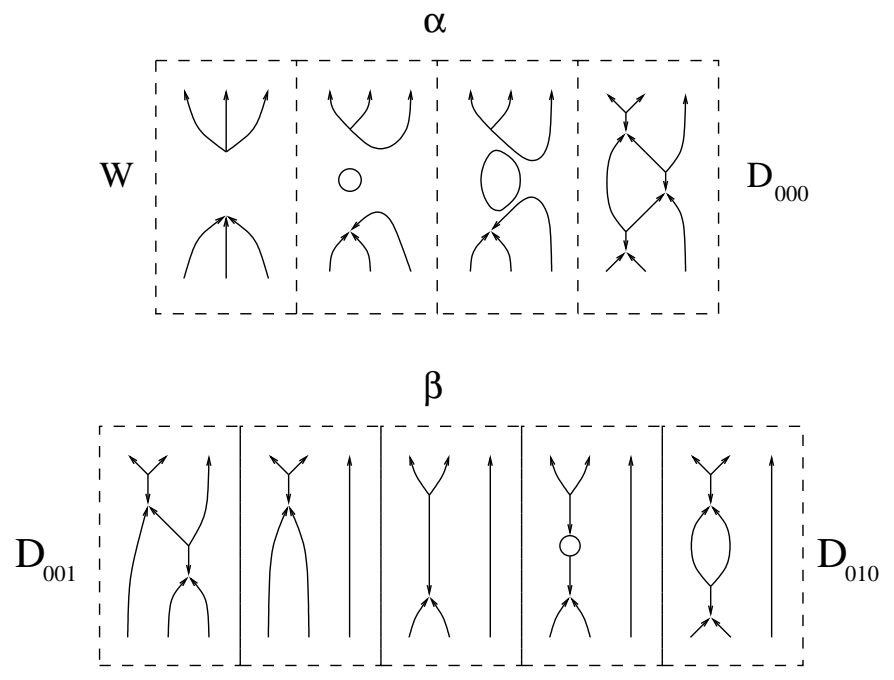

Figure 45: Cobordisms $\alpha$ and $\beta$

Let $Y_{2}$ be the subcomplex of $\mathcal{F}(D)$ generated by the subcomplex of $\mathcal{F}\left(D_{000}\right)$ isomorphic to $\mathcal{F}\left(D_{011}\right)$ via the inclusion induced by cobordism $\gamma$ in Figure 46 Let $Y_{3}$ be the subcomplex of $\mathcal{F}(D)$ generated by the subcomplex of $\mathcal{F}\left(D_{010}\right)$ isomorphic to $\mathcal{F}\left(D_{011}\right)$ via the inclusion induced by cobordism $\delta$ in Figure 46

Proposition $12 \mathcal{F}(D) \cong Y_{1} \oplus Y_{2} \oplus Y_{3}$. The complexes $Y_{2}$ and $Y_{3}$ are contractible. The complexes $Y_{1}$ and $Q$ are isomorphic.

A similar decomposition establishes homotopy equivalence of $\mathcal{F}\left(D^{\prime}\right)$ and $Q$, and implies that $\mathcal{F}(D)$ and $\mathcal{F}\left(D^{\prime}\right)$ are homotopy equivalent complexes.

\section{$6 \quad$ Flag varieties}

To a closed web $\Gamma$ associate a commutative graded ring $R(\Gamma)$ with generators $X_{i}$, over all edges $i$ of $\Gamma$, and relations

$$
X_{i}+X_{j}+X_{k}=0, \quad X_{i} X_{j}+X_{i} X_{k}+X_{j} X_{k}=0, \quad X_{i} X_{j} X_{k}=0 .
$$



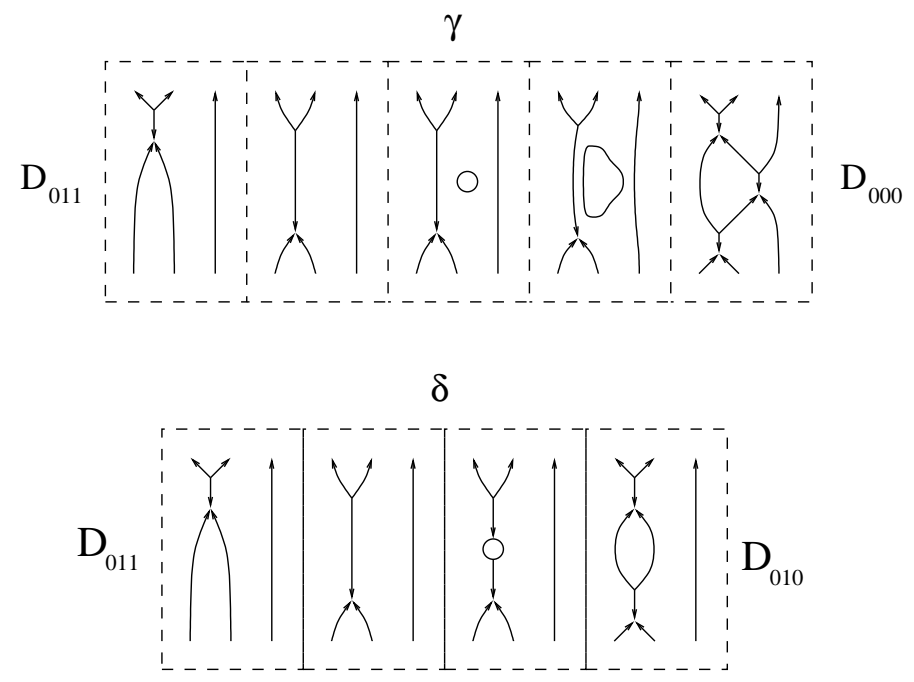

Figure 46: Cobordisms $\gamma$ and $\delta$

whenever edges $i, j, k$ meet at a vertex, and $X_{i}^{3}=0$ if $i$ is a closed loop. We set the degree of each $X_{i}$ to 2. Relations (13) are equivalent to the condition that every symmetric polynomial in $X_{i}, X_{j}, X_{k}$ vanishes. Relation $X_{i}^{3}=0$ for any non-closed edge $i$ follows from (13).

$\mathcal{F}(\Gamma)$ is a module over $R(\Gamma)$. The cobordism of merging a circle near edge $i$ with this edge induces a map $\mathcal{A} \otimes_{\mathbb{Z}} \mathcal{F}(\Gamma) \longrightarrow \mathcal{F}(\Gamma)$. Multiplication by $X \in \mathcal{A}$ is then an endomorphism $X_{i}$ of $\mathcal{F}(\Gamma)$ of degree 2. These endomorphisms satisfy relations (13) and make $\mathcal{F}(\Gamma)$ a module over $R(\Gamma)$.

If $\Gamma$ can be reduced to a circle by removing digon faces, see example in Figure 5 left (we call such $\Gamma$ a digon web), then $\mathcal{F}(\Gamma)$ is a free module over $R(\Gamma)$ of rank one. If $\Gamma$ is the cube web, see Figure 5 right, then $\mathcal{F}(\Gamma)$ is not free over $R(\Gamma)$.

Let $V$ be a three-dimensional complex vector space equipped with a hermitian form. Let $P(V)$ be the projective space of complex lines in $V$. To $V$ and $\Gamma$ we associate a topological space $V(\Gamma)$, a subspace of $P(V)^{\times E}$, where $E$ is the set of edges of $\Gamma$. A set of lines $\left\{L_{a}\right\}_{a \in E}, L_{a} \subset V$, is in $V(\Gamma)$ iff $L_{a}$ and $L_{b}$ are orthogonal whenever $a$ and $b$ share a common vertex.

The inclusion $V(\Gamma) \subset P(V)^{\times E}$ induces a homomorphism of cohomology rings $H\left(P(V)^{\times E}, \mathbb{Z}\right) \rightarrow H(V(\Gamma), \mathbb{Z})$ which factors through to a homomorphism $u$ : $R(\Gamma) \rightarrow H(V(\Gamma), \mathbb{Z})$. If $\Gamma$ is a digon web, $u$ is a ring isomorphism. We don't know if $u$ is an isomorphism for any $\Gamma$. 
If $\Gamma$ is a digon web,

$$
\mathcal{F}(\Gamma) \cong \mathrm{H}^{*}(V(\Gamma), \mathbb{Z})\left\{-\frac{n}{2}-2\right\}
$$

\section{References}

[1] B. Bakalov and A. A. Kirillov, Jr. Lectures on tensor categories and modular functors. University Lecture Series 21. AMS, Providence, RI, 2001. MathReview

[2] P. Freyd, D. Yetter, J. Hoste, W. B. R. Lickorish, K. Millett, and A. Ocneanu. A new polynomial invariant of knots and links. Bull. AMS (N.S.), 12(2):239-246, 1985. MathReview

[3] M. Jacobsson. An invariant of link cobordisms from Khovanov's homology theory. arXiv:math.GT/0206303

[4] M. Khovanov. Categorifications of the colored Jones polynomial. arXiv:math.QA/0302060

[5] M. Khovanov. An invariant of tangle cobordisms. arXiv:math.QA/0207264

[6] M. Khovanov. A categorification of the Jones polynomial. Duke Math J., 101(3):359-426, 2000. MathReview

[7] D. Kim. Graphical calculus of quantum Lie algebra representations. $\mathrm{PhD}$ thesis, University of California, Davis, 2003.

[8] G. Kuperberg. Spiders for rank 2 Lie algebras. Comm. Math. Phys., 180(1):109151, 1996. MathReview

[9] T. T. Q. Le. Integrality and symmetry of quantum link invariants. Duke Math. J., 102:273-306, 2000. MathReview

[10] H. Murakami, T. Ohtsuki, and S. Yamada. Homfly polynomial via an invariant of colored plane graphs. Enseign. Math. (2), 44:325-360, 1998. MathReview

[11] P. Ozsváth and Z. Szabó. Holomorphic disks and knot invariants. Adv. Math. 186(1):58-116, 2004. MathReview

[12] J. Rasmussen, Floer homology and knot complements. arXiv:math.GT/0306378

[13] N. Reshetikhin and V. Turaev. Ribbon graphs and their invariants derived from quantum groups. Comm. Math. Phys., 127:1-26, 1990. MathReview

Department of Mathematics, University of California, Davis CA 95616, USA

Email: mikhail@math.ucdavis.edu

Received: 26 May 2003 Revised: 7 October 2004 